\title{
Unprecedented Syndioselectivity and Syndiotactic Polyolefin Melting Temperature: Polypropylene and Poly(4-methyl-1-pentene) from a Highly Active, Sterically Expanded $\eta^{1}$-Fluorenyl- $\eta^{1}$-Amido Zirconium Complex
}

Levi J. Irwin and Stephen A. Miller*

Department of Chemistry, Texas A\&M University, College Station, Texas 77843-3255

\section{Supporting Information}

Supporting Information Available: Polymerization procedures and ${ }^{13} \mathrm{C}$ NMR spectra of the syndiotactic polymer samples reported in Table 1 and Table 2 as well as DSC thermograms.

\section{Table of Contents}

General Considerations, Instrumentation, and Polymerization Procedures............................ S2

${ }^{13} \mathrm{C}$ NMR Spectra of $s$-PP Samples Reported in Table 1........................................ S3

DSC Calibration Using a Certified Indium Standard............................................S8

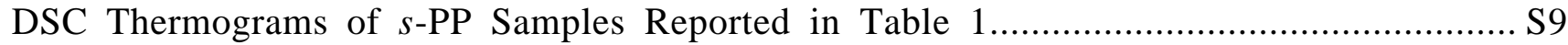

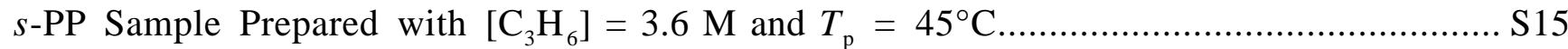

${ }^{13} \mathrm{C}$ NMR Spectra of $s$-P4M1P Samples Reported in Table 2 ......................................S16

DSC Thermograms of $s$-P4M1P Samples Reported in Table 2 2....................................S18

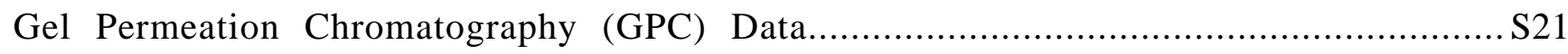

Demonstration of Thermal Stability of 3/MAO................................................... S31

Ethylene vs. Propylene Homopolymerization Activity Comparison with 1-5/MAO....................S32

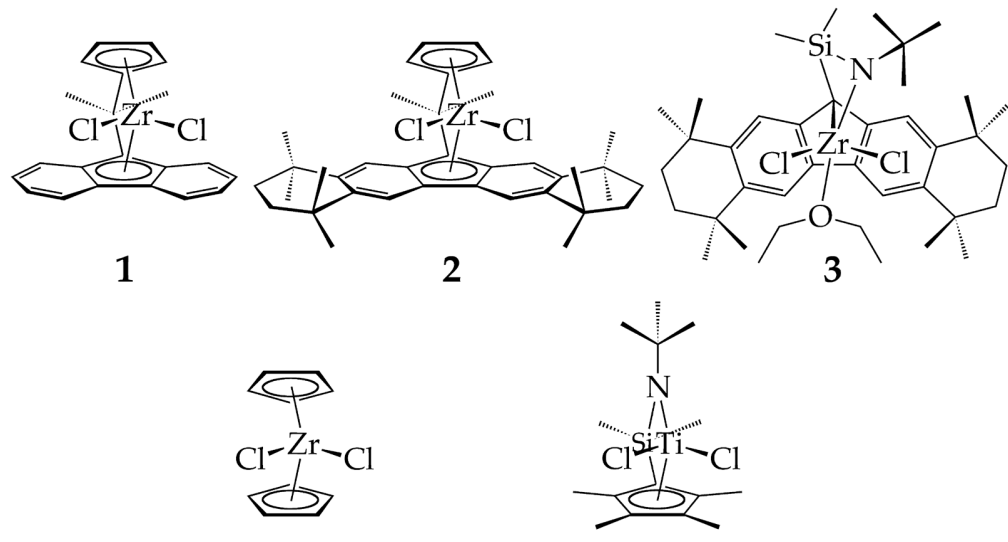


General Considerations and Instrumentation. ${ }^{13} \mathrm{C}$ NMR spectra of the syndiotactic polymers were recorded at $120^{\circ} \mathrm{C}$ on a Unity-Inova NMR operating at $74.42 \mathrm{MHz}$ in either 1,1,2,2-tetrachloroethane- $d_{2}$ or a $1: 1$ mixture of 1,2,4-trichlorobenzene and 1,1,2,2tetrachloroethane- $d_{2}$. The polypropylene pentad distributions were determined by integration of the resolved peaks in the methyl region (19-22 ppm) of the ${ }^{13} \mathrm{C}$ NMR spectra obtained. ${ }^{1}$ A 78 degree pulse was employed with broadband decoupling. The delay time was set to 3 seconds and a minimum of 18,000 scans were collected.

Polymer melting temperatures were determined on a Perkin Elmer Differential Scanning Calorimeter (DSC) Pyris 1 equipped with an Intracooler 2P, interfaced via the Pyris Thermal Analysis Software suite version 5.00.002. The instrument was calibrated by subjecting a certified Perkin Elmer indium standard (P-E P/N 0319-0033, Lot Code \# v-361) to conditions identical to the experimental runs. Polypropylene melting points were determined using a heating/cooling ramp of $10^{\circ} \mathrm{C} / \mathrm{min}$. from $25^{\circ} \mathrm{C}$ to $200^{\circ} \mathrm{C}$ back to $25^{\circ} \mathrm{C}$. Poly(4-methyl-1-pentene) melting points were determined using a heating/cooling ramp of $10^{\circ} \mathrm{C} / \mathrm{min}$. from $25^{\circ} \mathrm{C}$ to $300^{\circ} \mathrm{C}$ back to $25^{\circ} \mathrm{C}$. In each case the results of the second run are reported and the maximum of the melting endotherm taken as the melting point.

Toluene was prepared by distilling from elemental sodium under an atmosphere of nitrogen and stored in a Straus flask in the glove box until needed. Propylene from Scott Specialty Gases (>99.5\%) was used following passage through a Matheson 6410 drying system equipped with an OXYSORB ${ }^{\mathrm{TM}}$ column. 4-methyl-1-pentene was acquired from TCI with a purity of $97 \%$. The monomer was dried by stirring over $\mathrm{CaH}_{2}$, degassed via three successive freeze-pump-thaw cycles, and then vacuum transferred into a Straus flask to be stored in the glove box until needed. Methylaluminoxane (MAO) was acquired from Albemarle as a $30 \mathrm{wt} \%$ solution in toluene and prepared by drying under high vacuum at $70^{\circ} \mathrm{C}$ for 3 days.

Stock solutions of each catalyst were prepared by placing $0.100 \mathrm{~g}$ of $\mathbf{1}, 0.200 \mathrm{~g}$ of $\mathbf{2}$, and $0.250 \mathrm{~g}$ of $\mathbf{3}$ in their respective $100 \mathrm{~mL}$ volumetric flasks and adding toluene. The stock solutions were then diluted using standard volumetric procedures in order to produce the following concentrations: $[\mathbf{1}]=1.62 \times 10^{-3} \mathrm{M},[2]=1.53 \times 10^{-3} \mathrm{M}$, and $[3]=1.66 \times 10^{-3}$ M. Polymerizations were initiated by injecting $1.0 \mathrm{~mL}$ of catalyst solution into the reactor, as described below.

Polymerization Procedure. CAUTION: All polymerizations should be carried out in a fume hood behind a blast shield. Propylene polymerizations were carried out in an $85 \mathrm{~mL}$ glass Lab-Crest ${ }^{\circledR}$ (Andrews Glass Co.) cylindrical polymerization reactor equipped with a 2 inch cylindrical stir bar able to provide ample surface agitation while stirring. The apparatus was brought into the box where the reactor was charged with solid MAO (1000 equivalents, prepared as described above) and then assembled. The reactor was purged with propylene gas for 3 minutes before being sealed; then $30 \mathrm{~mL}$ of propylene were condensed in at $0^{\circ} \mathrm{C}$ and 120 psi. The system was then allowed to equilibrate for 15 minutes in either an

(1) a) Busico, V.; Cipullo, R.; Corradini, P.; Landriani, L.; Vacatello, M.; Segre, A. L. Macromolecules 1995, 28, 1887-1892. b) Busico, V.; Cipullo, R.; Monaco, G.; Vacatello, M. Macromolecules 1997, 30 , 6251-6263. ice/salt bath $\left(T_{\mathrm{p}}=-15^{\circ} \mathrm{C}\right)$, an ice bath $\left(T_{\mathrm{p}}=0^{\circ} \mathrm{C}\right)$, or a room temperature water bath $\left(T_{\mathrm{p}}=25^{\circ} \mathrm{C}\right)$. After thermal equilibration, $1.0 \mathrm{~mL}$ of the catalyst solution was injected into the reactor via a $2.5 \mathrm{~mL}$ Hamilton Gastight syringe. The polymerizations were run for 30 minutes if $T_{\mathrm{p}}=-15^{\circ} \mathrm{C}$, 5 minutes if $T_{\mathrm{p}}=0^{\circ} \mathrm{C}$, or 1 minute if $T_{\mathrm{p}}=25^{\circ} \mathrm{C}$. After the prescribed time the vessel was quickly vented and the contents combined with $200 \mathrm{~mL}$ of a $5 \%(\mathrm{v} / \mathrm{v})$ aqueous concentrated $\mathrm{HCl} /$ methanol solution. The precipitated polymer was collected by filtration and washed 5 times (30 $\mathrm{mL}$ each) with fresh methanol before being dried under high vacuum.

The polymerization of 4-methyl-1-pentene was accomplished by bringing the reactor into the glove box and charging it with 1000 eq. MAO followed by $25 \mathrm{~mL}$ of 4-methyl-1-pentene. The reactor was then sealed, brought out of the glove box, and placed in a bath at the appropriate temperature (as described above). The system was allowed to thermally equilibrate for 15 minutes before the catalyst solution was injected. After the prescribed time the polymerization was quenched by injection of $5 \mathrm{~mL}$ of a $5 \%$ (v/v) solution of aqueous concentrated $\mathrm{HCl} / \mathrm{methanol.} \mathrm{The}$ contents of the reactor were then combined with an additional $150 \mathrm{~mL}$ of $5 \%(\mathrm{v} / \mathrm{v})$ aqueous concentrated $\mathrm{HCl} /$ methanol and vigorously stirred. The precipitated polymer was collected by filtration and washed 5 times (30 $\mathrm{mL}$ each) with fresh methanol before being dried under high vacuum. 


\section{${ }^{13}$ C NMR Spectra of $s$-PP Samples Reported in Table 1}

(Insets are enlargements of the methyl region.)

Table 1. MAO-activated propylene polymerization results for 1-5. ${ }^{a}$

\begin{tabular}{|c|c|c|c|c|c|c|c|c|c|c|c|c|c|c|c|}
\hline Entry & $\begin{array}{c}\text { Catalyst } \\
(\mu \mathrm{mol})\end{array}$ & $\begin{array}{c}T_{\mathrm{p}} \\
\left({ }^{\circ} \mathrm{C}\right)\end{array}$ & $\begin{array}{l}\text { Yield } \\
(\mathrm{g})\end{array}$ & Activity $^{\mathrm{b}}$ & $\begin{array}{c}T_{\mathrm{m}}^{\mathrm{c}} \\
\left({ }^{\circ} \mathrm{C}\right)\end{array}$ & $\begin{array}{c}{[r r r r]^{\mathrm{d}}} \\
\%\end{array}$ & \multicolumn{3}{|c|}{ Injection 1} & \multicolumn{3}{|c|}{ Injection 2} & \multicolumn{3}{|c|}{ Average } \\
\hline 1 & $\mathbf{1}(1.62)$ & 0 & 0.32 & 2,400 & 151 & 91 & 209,379 & 84,326 & 2.4830 & 208,955 & 84,934 & 2.4602 & 209,200 & 84,600 & 2.47 \\
\hline 3 & $\mathbf{1}(1.62)^{\mathrm{f}}$ & 90 & 1.16 & 42,700 & 116 & 71 & 57,365 & 26,677 & 2.1504 & 57,433 & 26,822 & 2.1413 & 57,400 & 26,700 & 2.16 \\
\hline 4 & $2(1.53)$ & 0 & 0.23 & 1,830 & 157 & 96 & 498,685 & 194,851 & 2.5593 & 492,181 & 192,676 & 2.5544 & 495,400 & 193,800 & 2.56 \\
\hline 5 & $2(1.53)$ & 25 & 0.45 & 17,730 & 151 & 92 & 363,804 & 127,896 & 2.8445 & 361,684 & 126,070 & 2.8689 & 362,700 & 127,000 & 2.86 \\
\hline 7 & $3(1.66)$ & -15 & 1.08 & 1,310 & 165 & $>99$ & 58,551 & 26,074 & 2.2456 & 58,728 & 26,008 & 2.2581 & 58,600 & 26,000 & 2.25 \\
\hline 8 & $3(1.66)$ & 0 & 0.54 & 3,890 & 164 & 98 & 46,189 & 23,226 & 1.9887 & 46,296 & 23,519 & 1.9685 & 46,200 & 23,400 & 1.98 \\
\hline 9 & $3(1.66)$ & 25 & 1.07 & 38,670 & 157 & 96 & 35,732 & 16,341 & 2.1866 & 35,611 & 16,294 & 2.1855 & 35,700 & 16,300 & 2.17 \\
\hline 10 & $3(1.66)^{f}$ & 90 & 1.14 & 41,100 & 128 & 82 & 14,461 & 7,478 & 1.9338 & 14,411 & 7,477 & 1.9274 & 14,400 & 7,500 & 1.93 \\
\hline 11 & $4(1.66)$ & 0 & 0.04 & 290 & am. & - & - & - & - & - & - & - & - & - & - \\
\hline 12 & $4(1.66)$ & 25 & 0.04 & 1,450 & am. & - & - & - & - & - & - & - & - & - & - \\
\hline
\end{tabular}

${ }^{a}$ Polymerization conditions: neat propylene $(30 \mathrm{~mL}) ; 1000$ eq. MAO; $30 \mathrm{~min}$. run at $T_{\mathrm{p}}=-15^{\circ} \mathrm{C} ; 5 \mathrm{~min}$. runs at $T_{\mathrm{p}}=0^{\circ} \mathrm{C} ; 1 \mathrm{~min}$. runs at $T_{\mathrm{p}}=25^{\circ} \mathrm{C}$. ${ }^{\mathrm{b}} \mathrm{kg}$ $\mathrm{PP} /(\mathrm{mol} \mathrm{M} \cdot \mathrm{h})$. ${ }^{\mathrm{c}}$ Unannealed, determined by DSC; am. = amorphous. ${ }^{\mathrm{d}}[\mathrm{rrrr}] \%$ determined by ${ }^{13} \mathrm{C}$ NMR. ${ }^{\mathrm{e}}$ Average of two injections vs. polystyrene. ${ }^{\text {f}}$ Polymerization conditions: $30 \mathrm{~mL} n$-heptane; 1000 eq. MAO; 120 psi propylene; $10 \mathrm{~min}$. pre-run equilibration; $1 \mathrm{~min}$. run.

Full pentad distribution for the polypropylene samples reported in Table 1

\begin{tabular}{cccccccccc}
\hline Entry & {$[\mathrm{mmmm}]$} & {$[\mathrm{mmmr}]$} & {$[\mathrm{rmmr}]$} & {$[\mathrm{mmrr}]$} & $\begin{array}{c}{[\mathrm{mmrm}]} \\
+[\mathrm{rrmr}]\end{array}$ & {$[\mathrm{mrmr}]$} & {$[\mathrm{rrrr}]$} & {$[\mathrm{rrm}]$} & {$[\mathrm{mrrm}]$} \\
\hline 1 & - & - & 1.0 & 2.5 & 1.0 & - & 91.4 & 4.1 & - \\
2 & - & - & 1.4 & 2.3 & 2.0 & - & 89.0 & 5.3 & - \\
3 & - & - & 1.9 & 4.8 & 7.5 & - & 71.0 & 14.4 & - \\
4 & - & - & - & - & 1.6 & - & 95.6 & 2.8 & - \\
5 & - & - & - & - & 3.4 & - & 92.1 & 4.5 & - \\
6 & - & - & 1.6 & 3.8 & 13.8 & 1.6 & 64.0 & 15.2 & - \\
7 & - & - & - & - & - & - & $99+$ & - & - \\
8 & - & - & - & - & 1.1 & - & 97.6 & 1.3 & - \\
9 & - & - & - & - & 1.9 & - & 96.1 & 2.1 & - \\
10 & - & - & 0.5 & 1.1 & 7.8 & - & 82.0 & 8.7 & - \\
13 & 0.4 & 5.0 & 6.5 & 13.2 & 21.1 & 11.1 & 21.5 & 18.1 & 2.9 \\
\hline
\end{tabular}


Entry 1: $[r r r r]=91 \%$

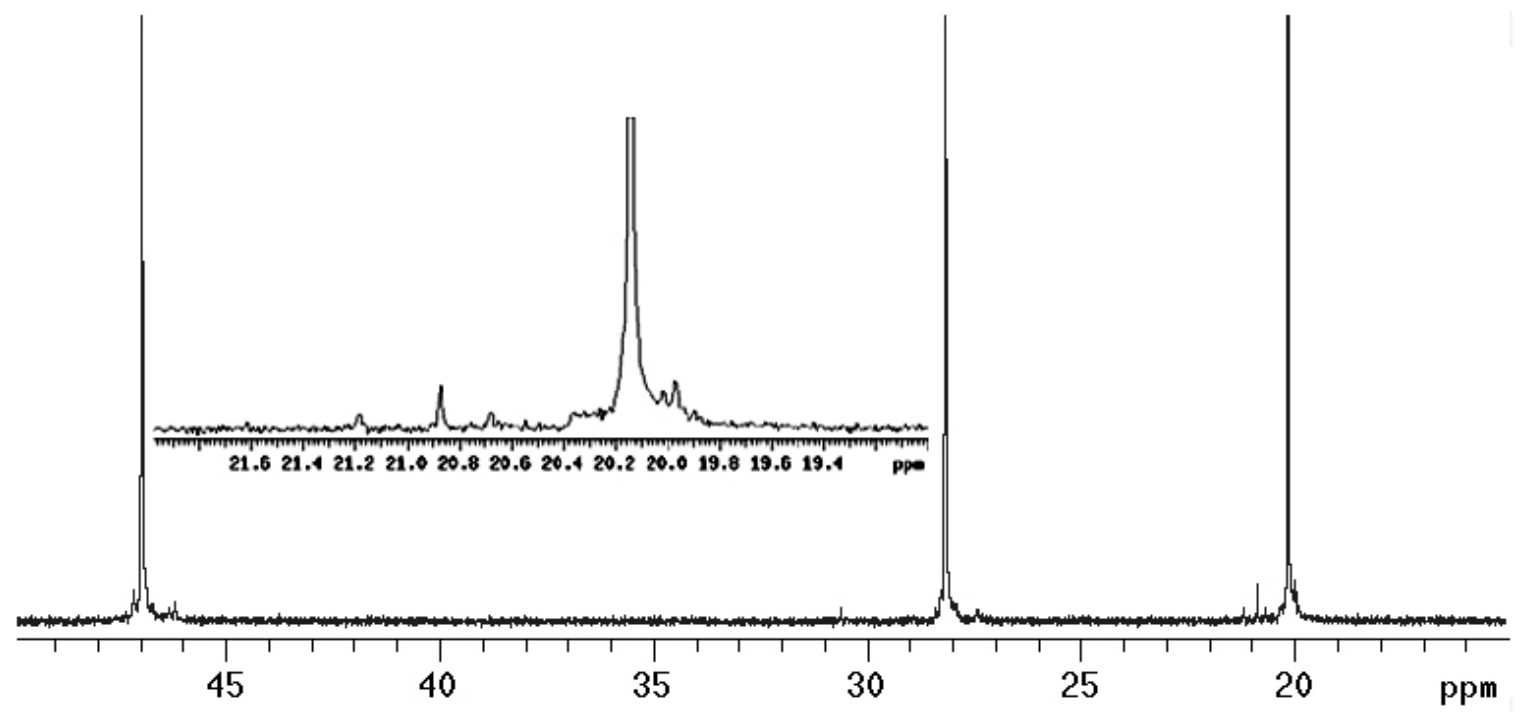

Entry 2: $[r r r r]=87 \%$

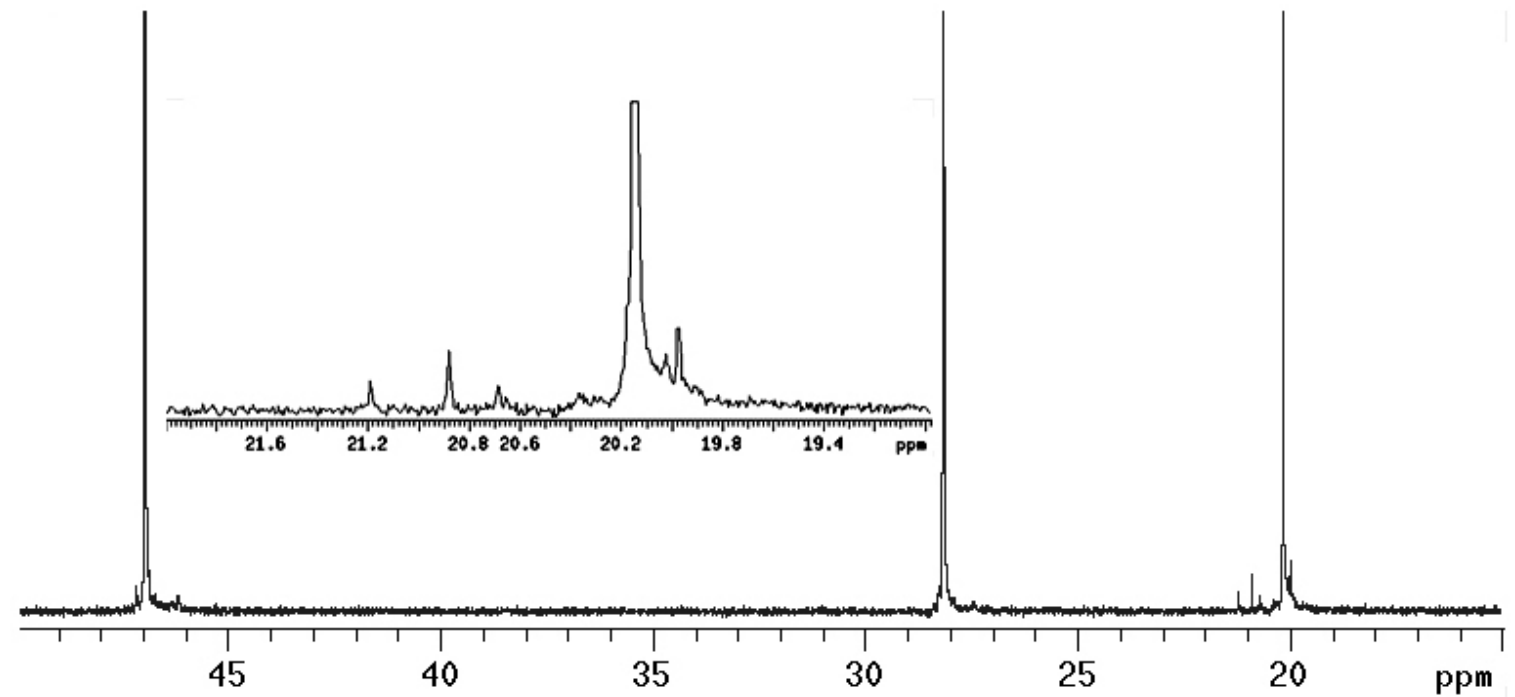

Entry 3: $[r r r r]=71 \%$

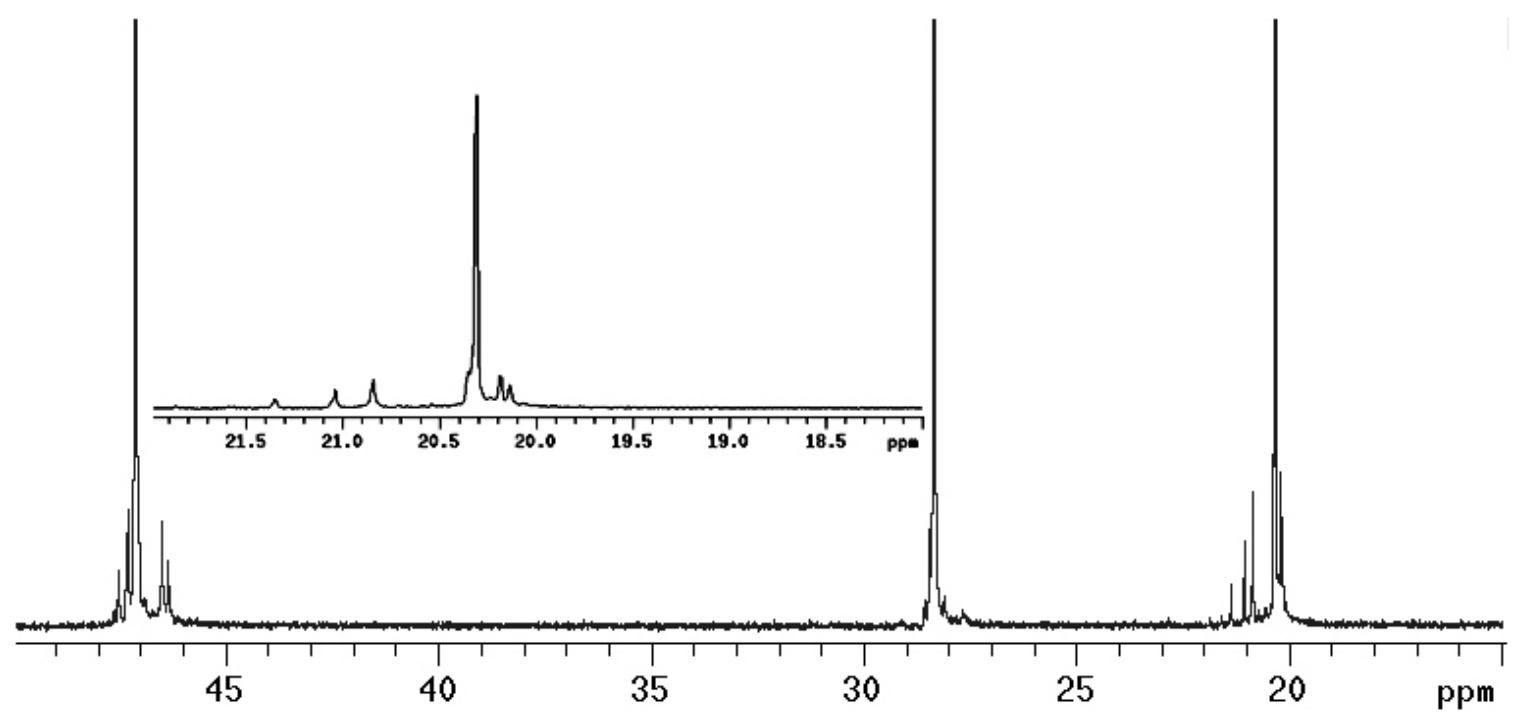


Entry 4: $[r r r r]=96 \%$

Even after 30,000 transients, the stereoerrors in this sample are difficult to see in the methyl region.

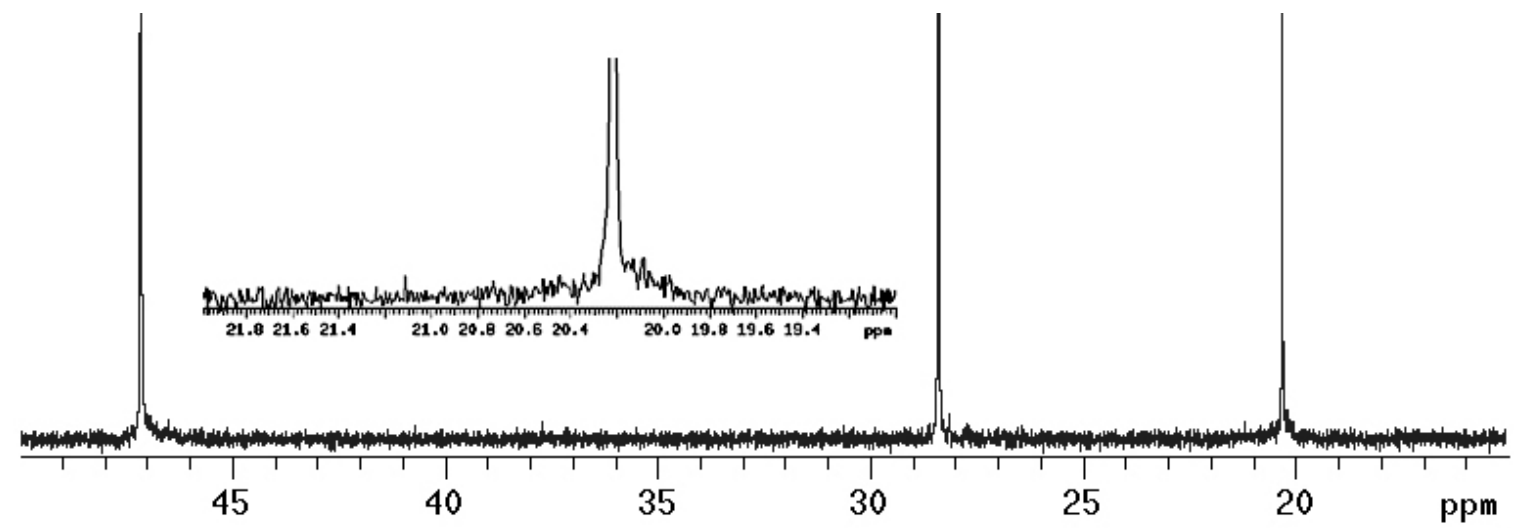

Entry 5: $[r r r r]=92 \%$

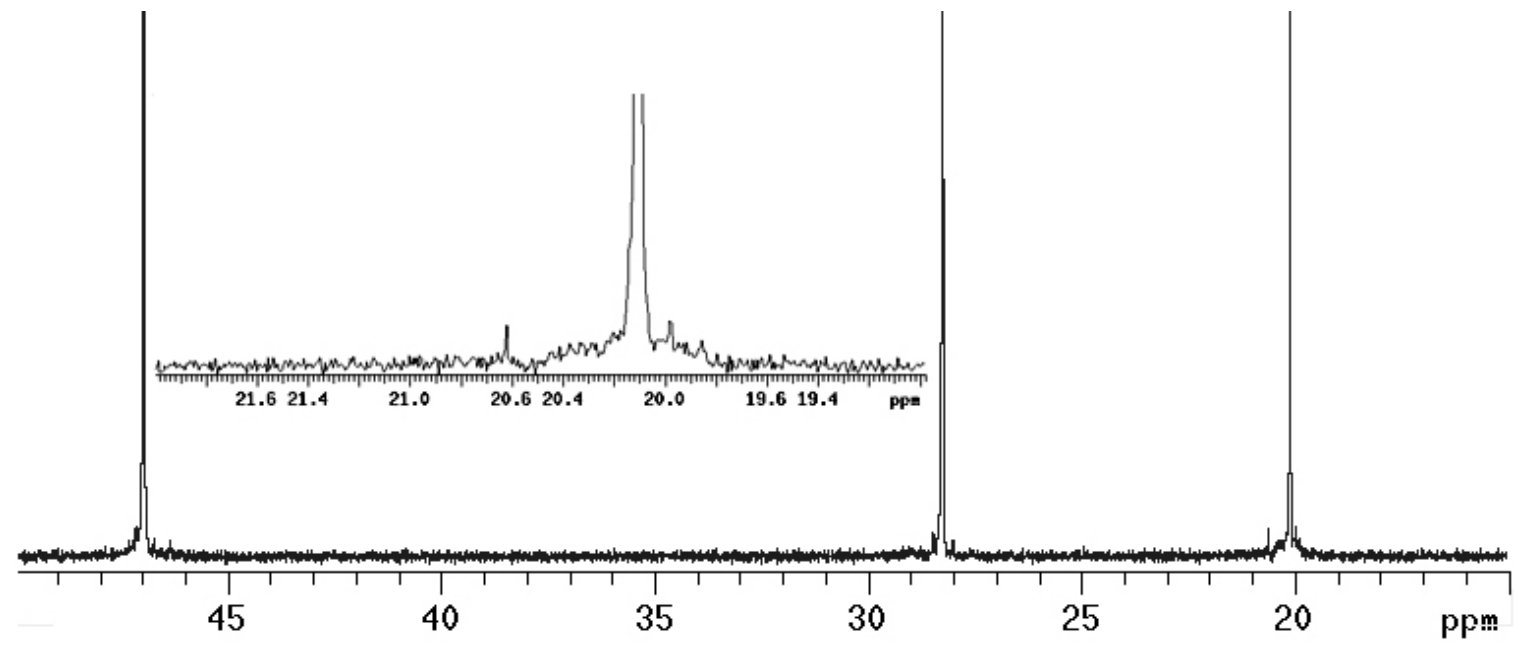

Entry 6: $[r r r r]=64 \%$

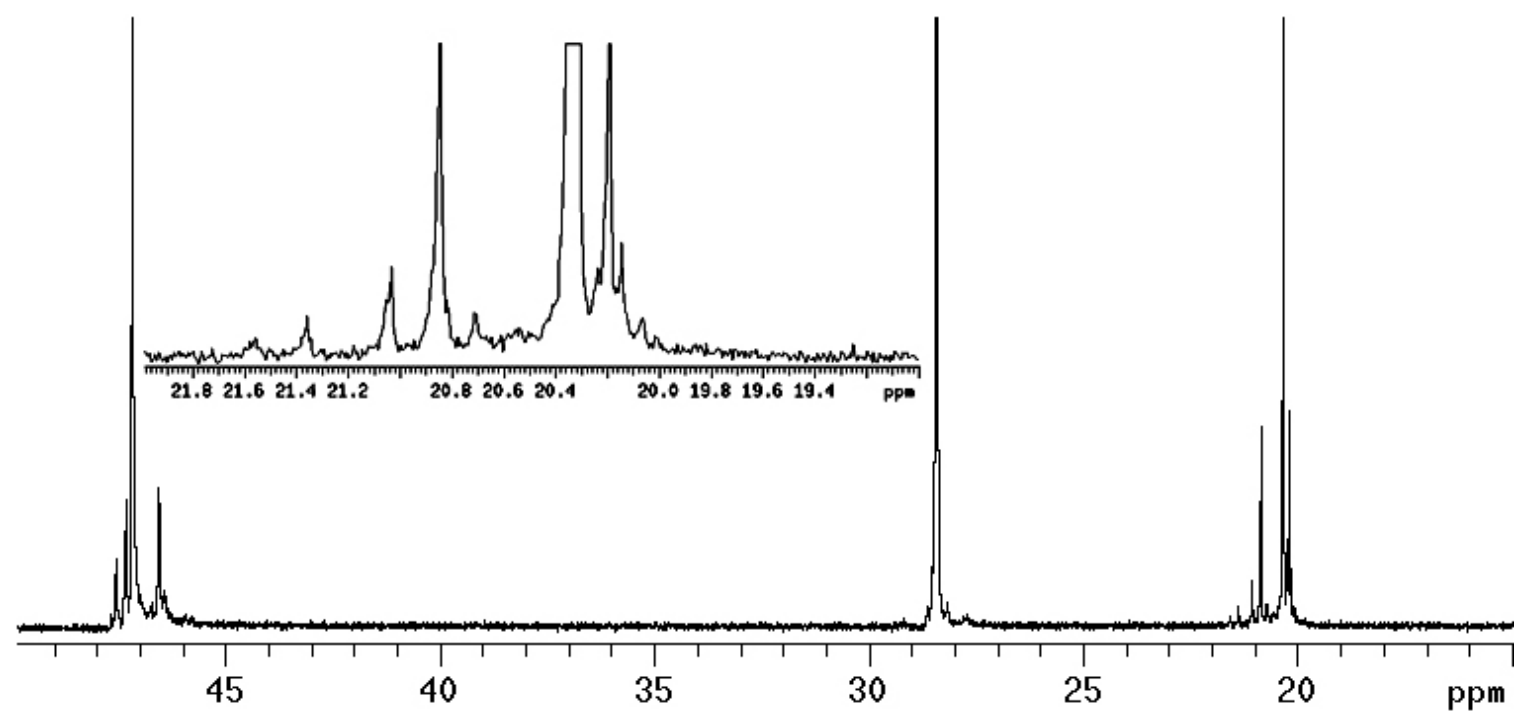


Entry 7: $[r r r r]>99 \%$

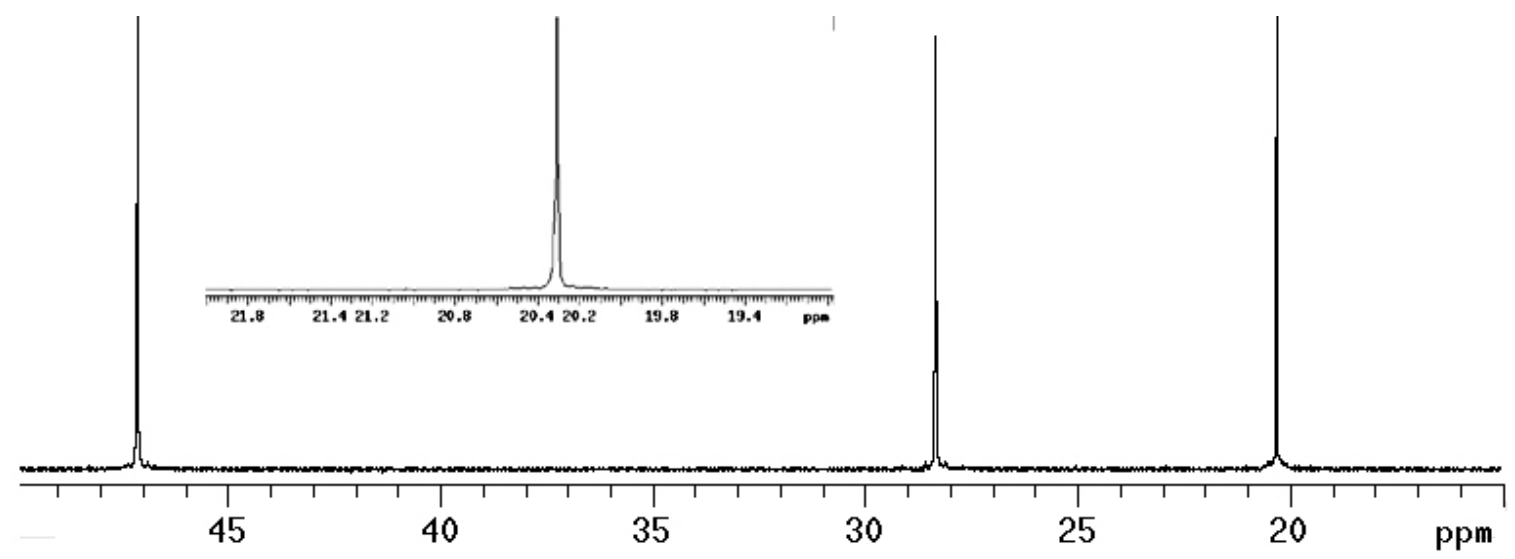

Entry 8: $[r r r r]=98 \%$

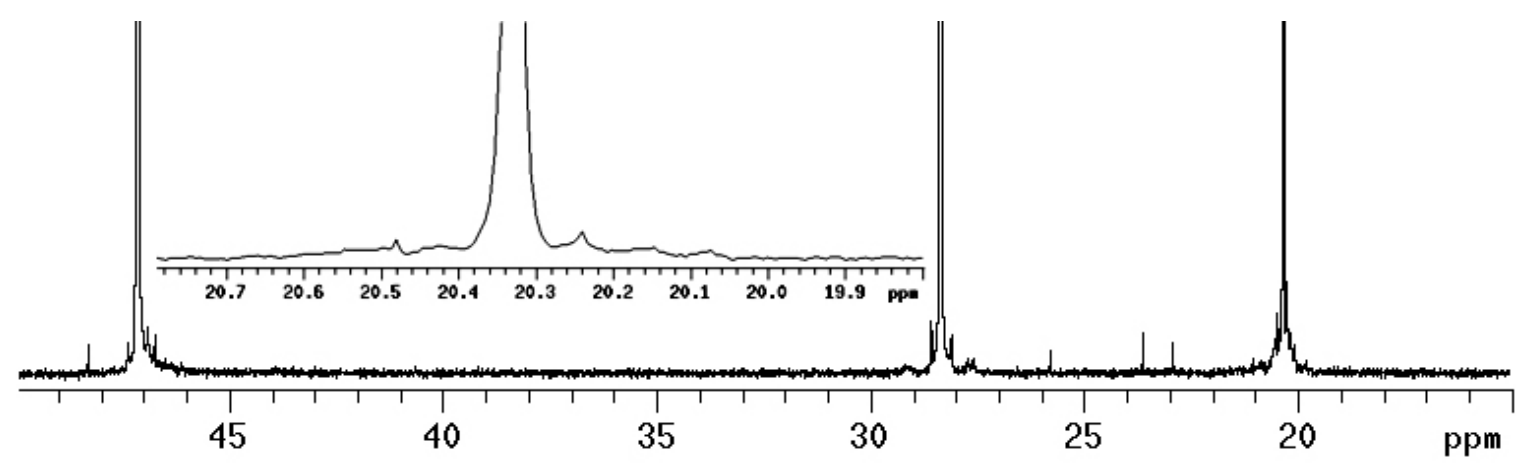

Entry 9: $[r r r r]=96 \%$

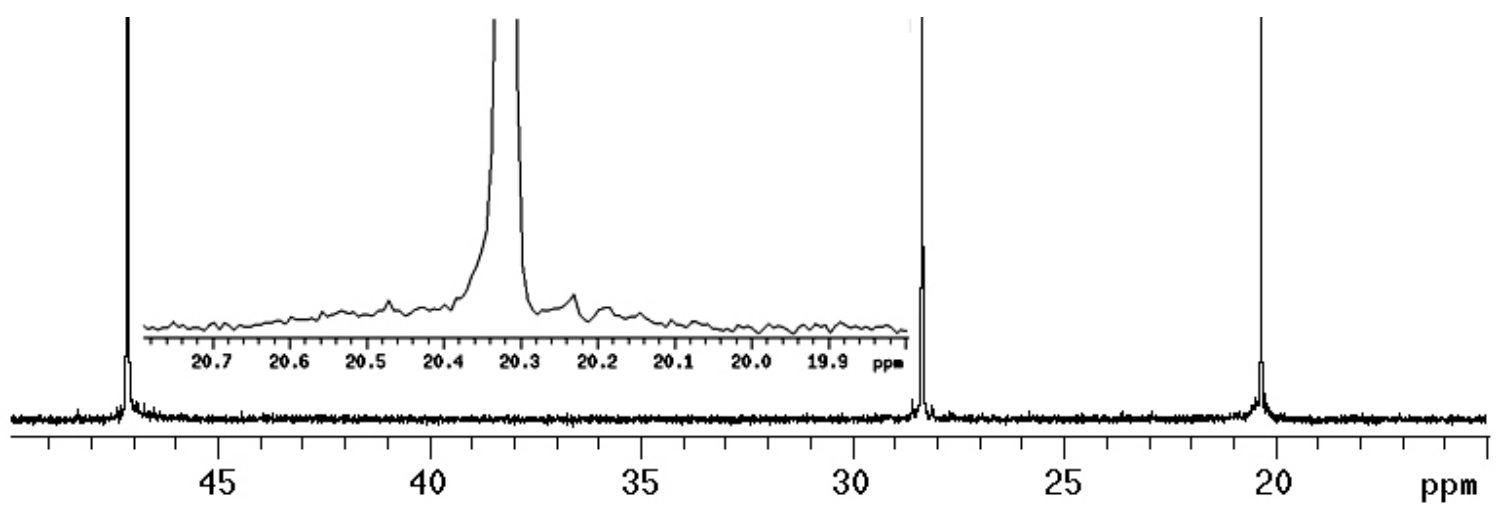


Entry 10: $[r r r r]=82 \%$

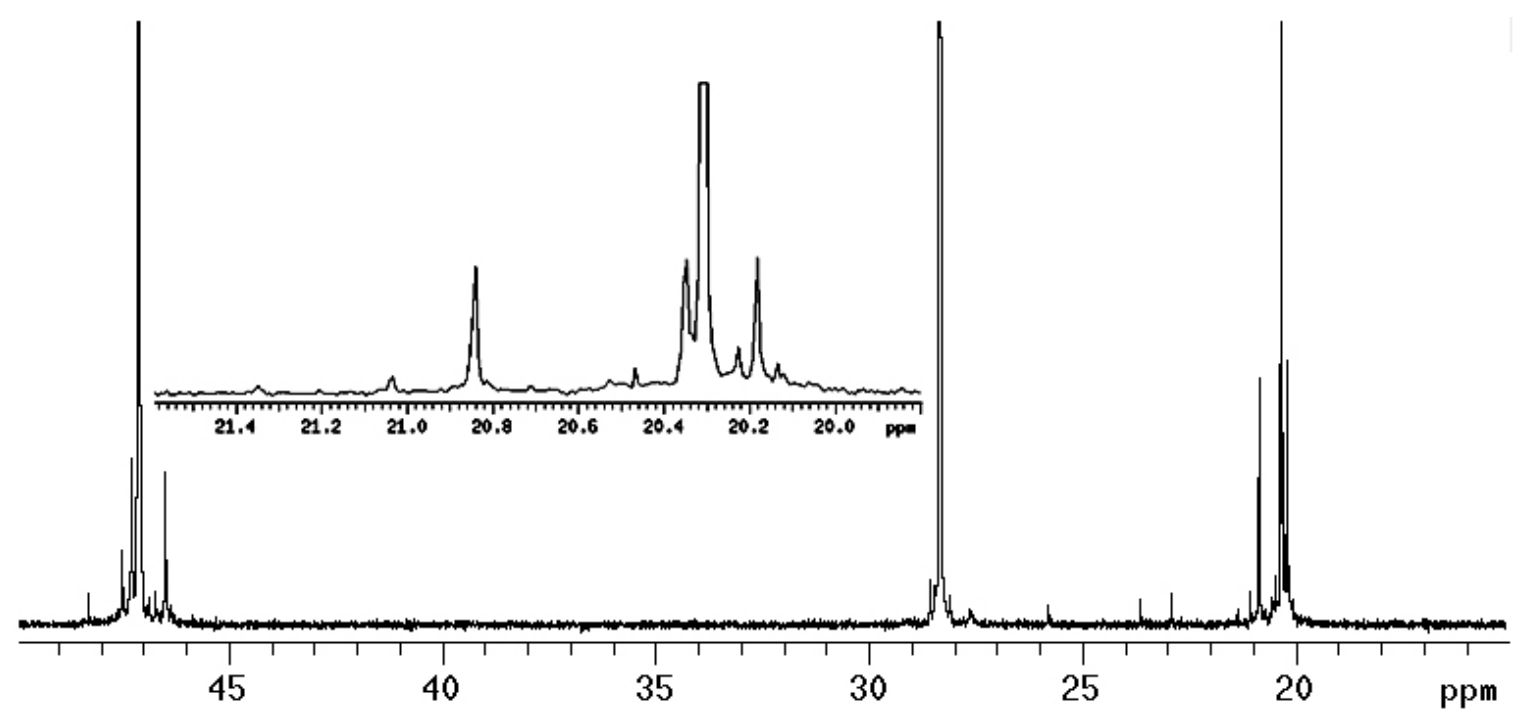

Entry 13: $[r r r r]=22 \%$

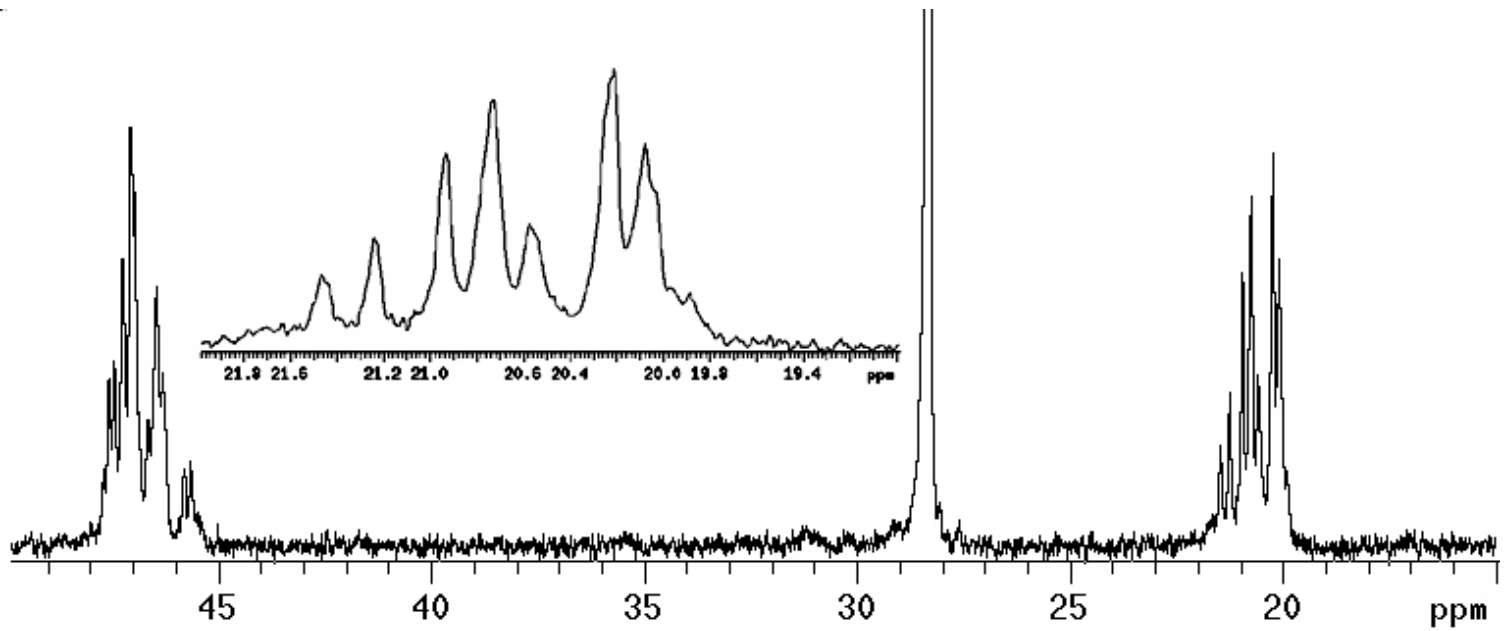




\section{DSC Calibration Using a Certified Indium Standard}

Onset measured $=156.57^{\circ} \mathrm{C}$

Expected $=156.5985^{\circ} \mathrm{C}($ see reference 2$)$

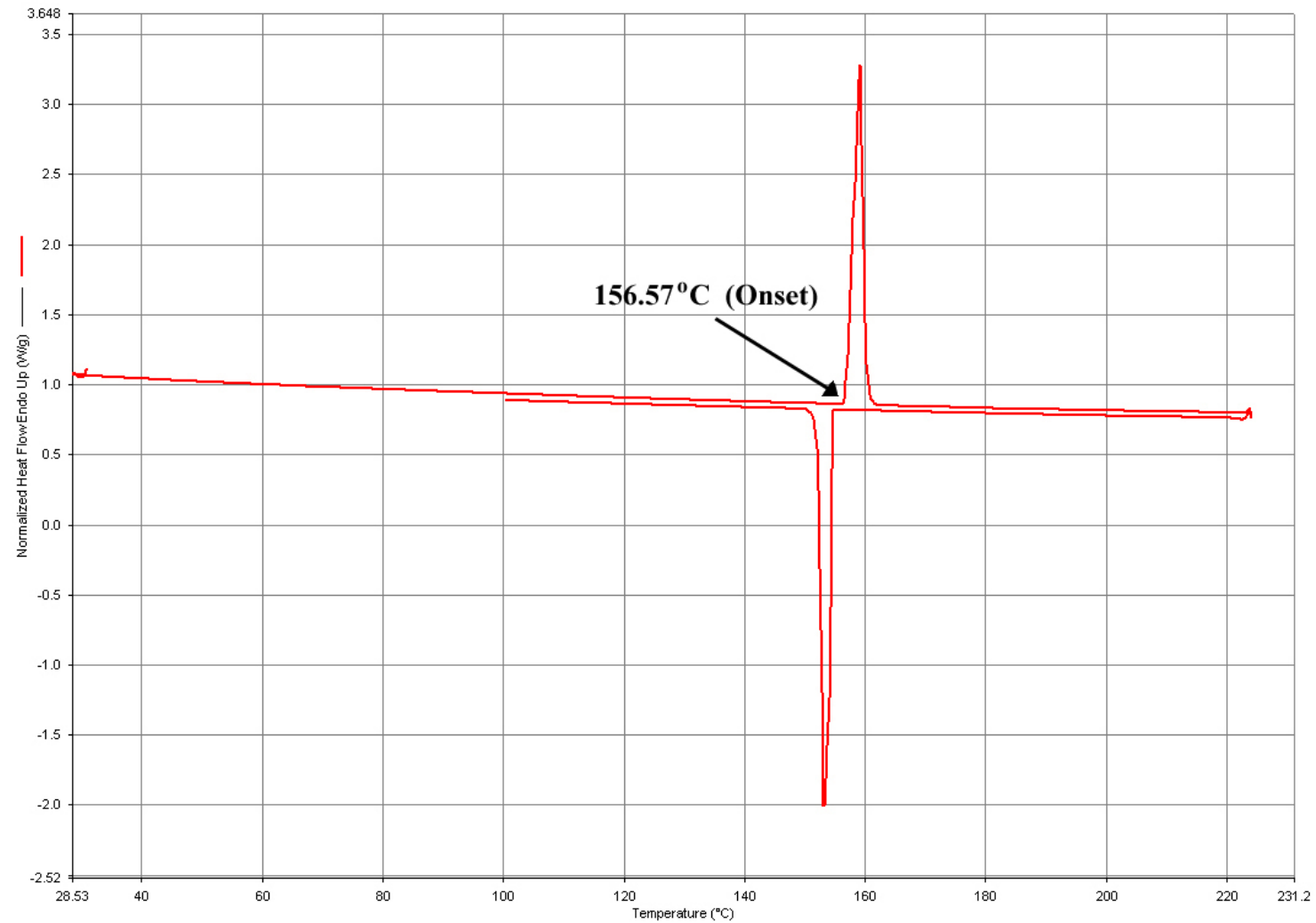

(2) Sarge, S. M.; Gmelin, E.; Höhne, G. W. H.; Cammenga, H. K.; Hemminger, W.; Eysel, W. Thermochim. Acta 1994, 247, $129-168$. 
DSC Thermograms of $s$-PP Samples Reported in Table 1

Entry 1: $T_{\mathrm{m}}=151^{\circ} \mathrm{C}$

(endotherm-down)

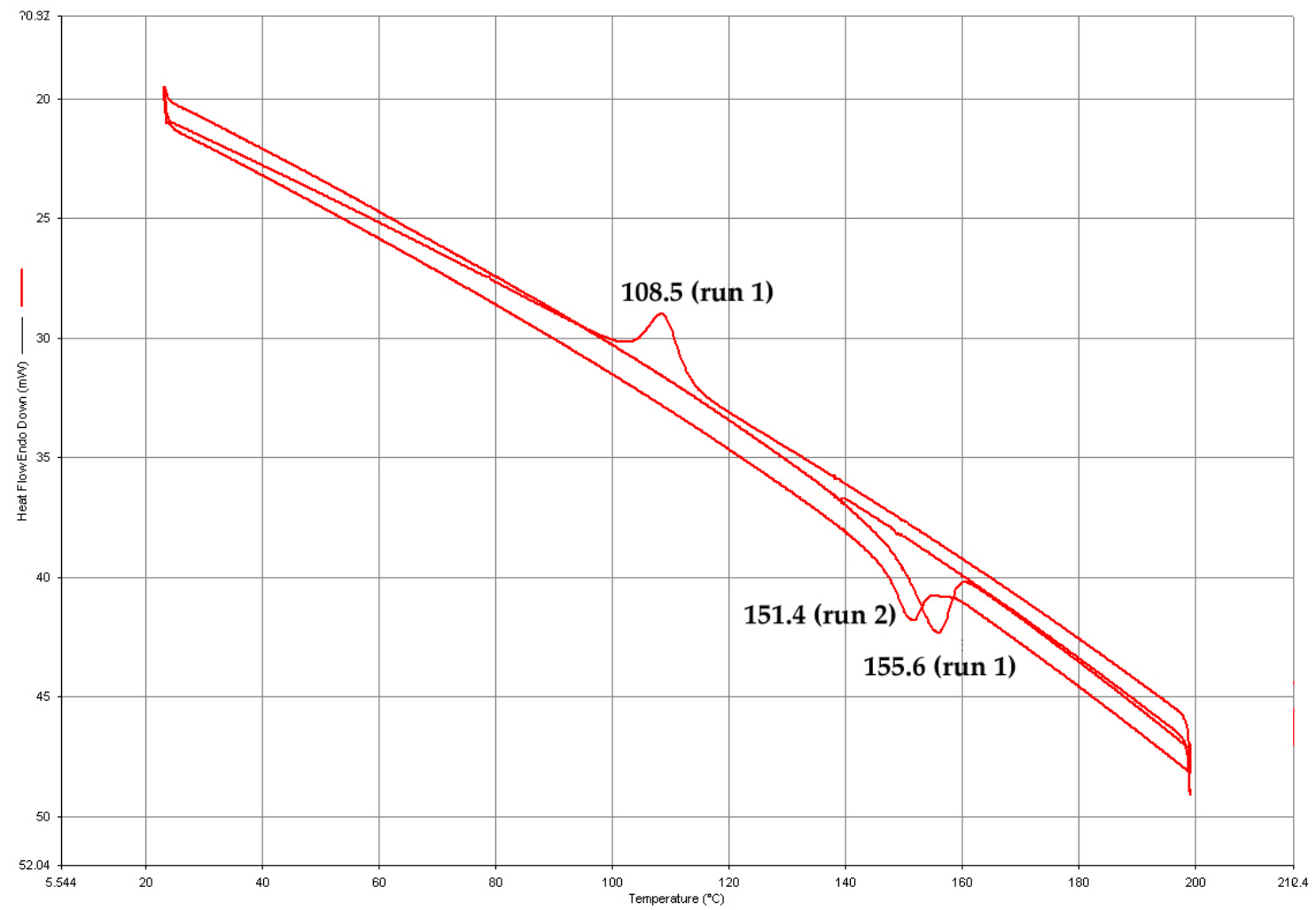

Entry 2: $T_{\mathrm{m}}=142^{\circ} \mathrm{C}$

(endotherm-up)

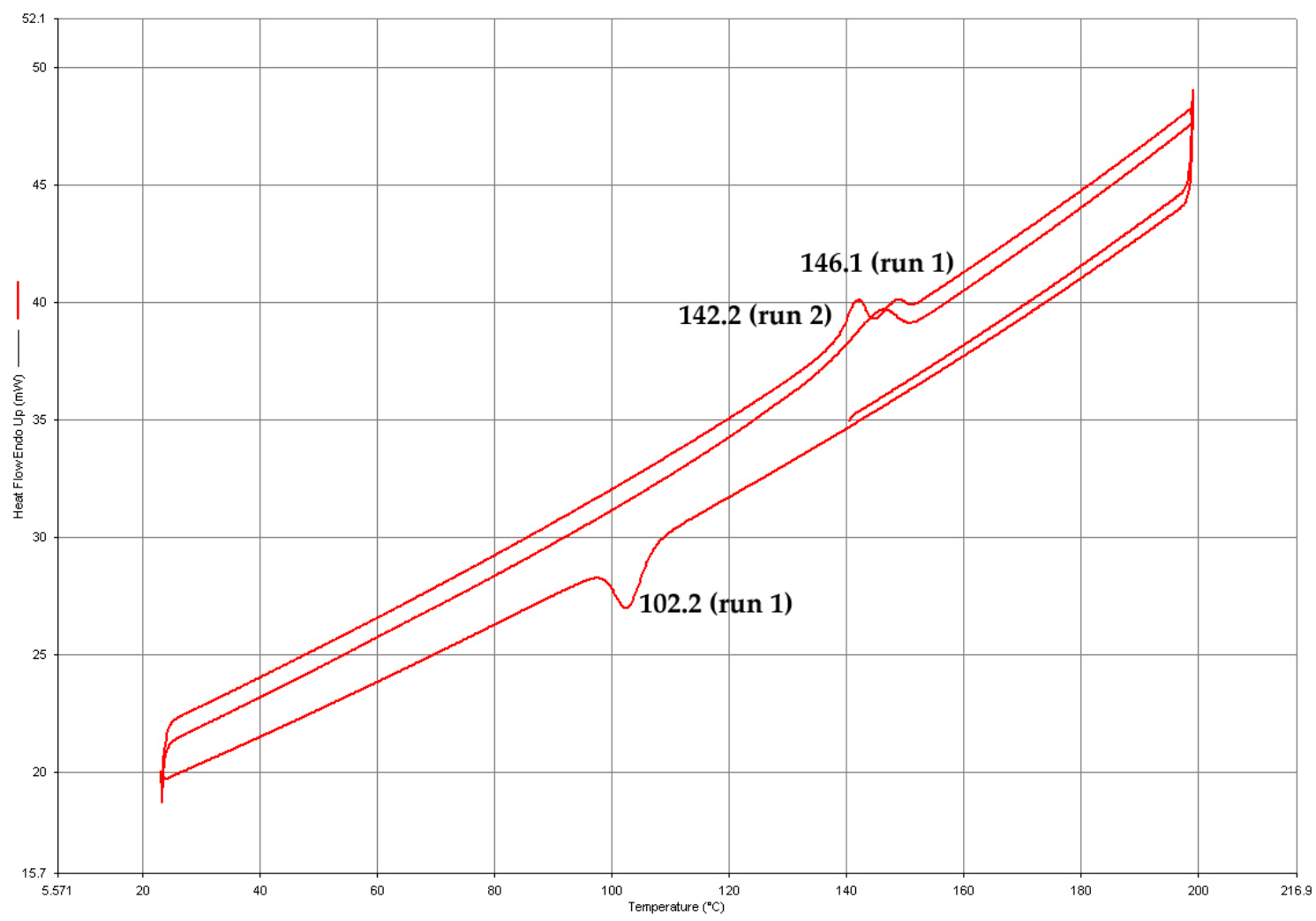


Entry 3: $T_{\mathrm{m}}=116^{\circ} \mathrm{C}$

(endotherm-down)

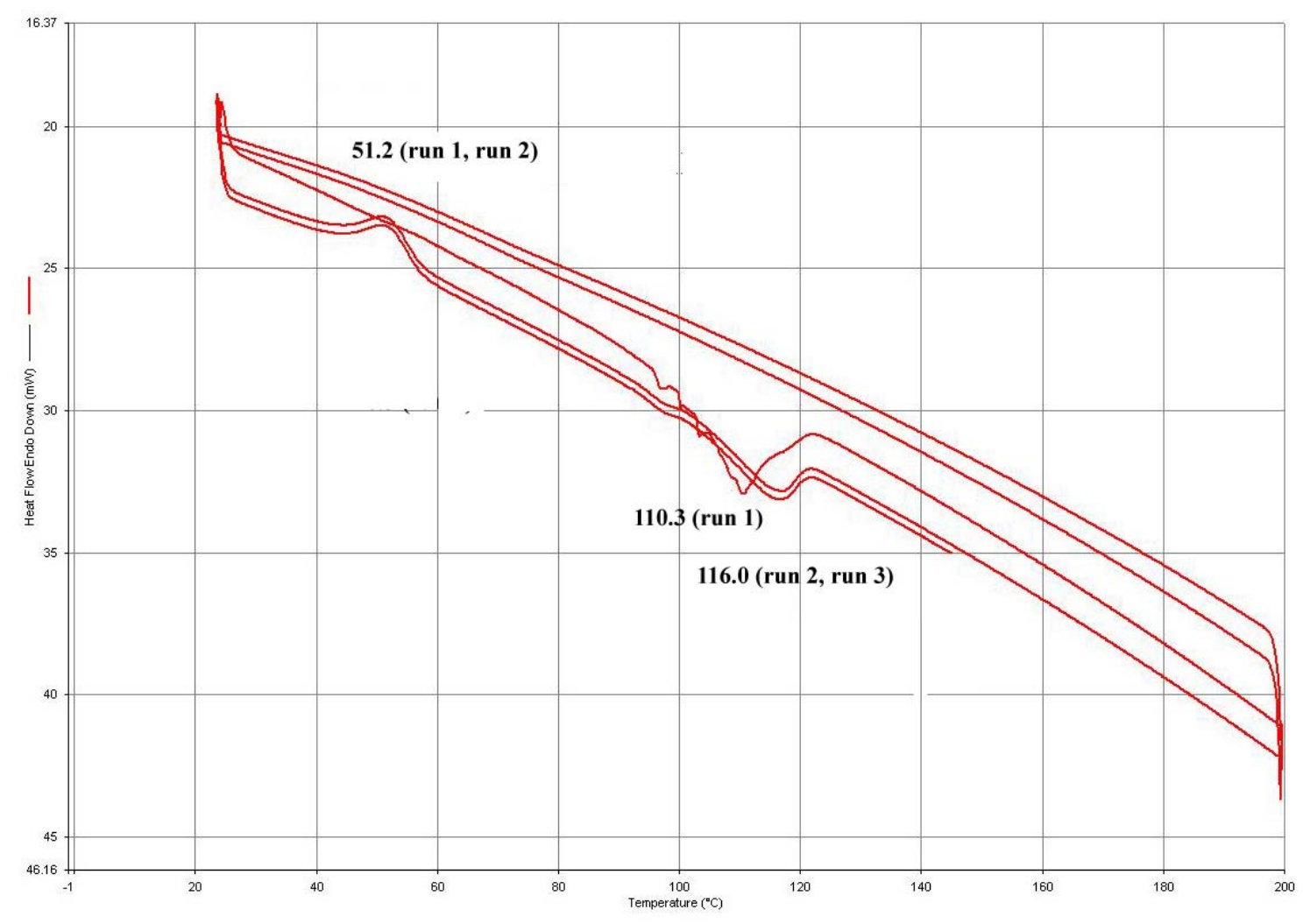

Entry 4: $T_{\mathrm{m}}=157^{\circ} \mathrm{C}$

(endotherm-down)

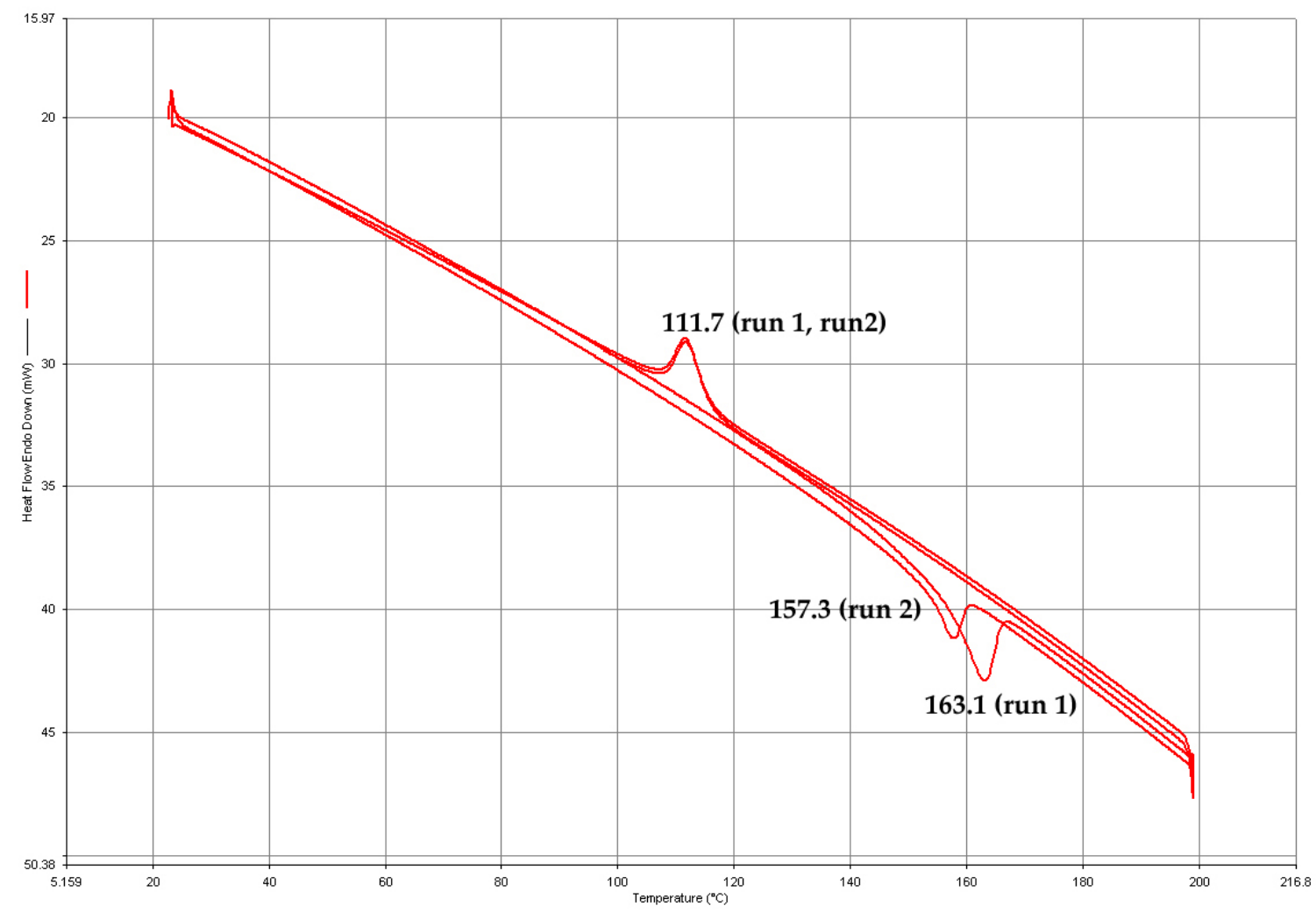


Entry 5: $T_{\mathrm{m}}=151^{\circ} \mathrm{C}$

(endotherm-up)

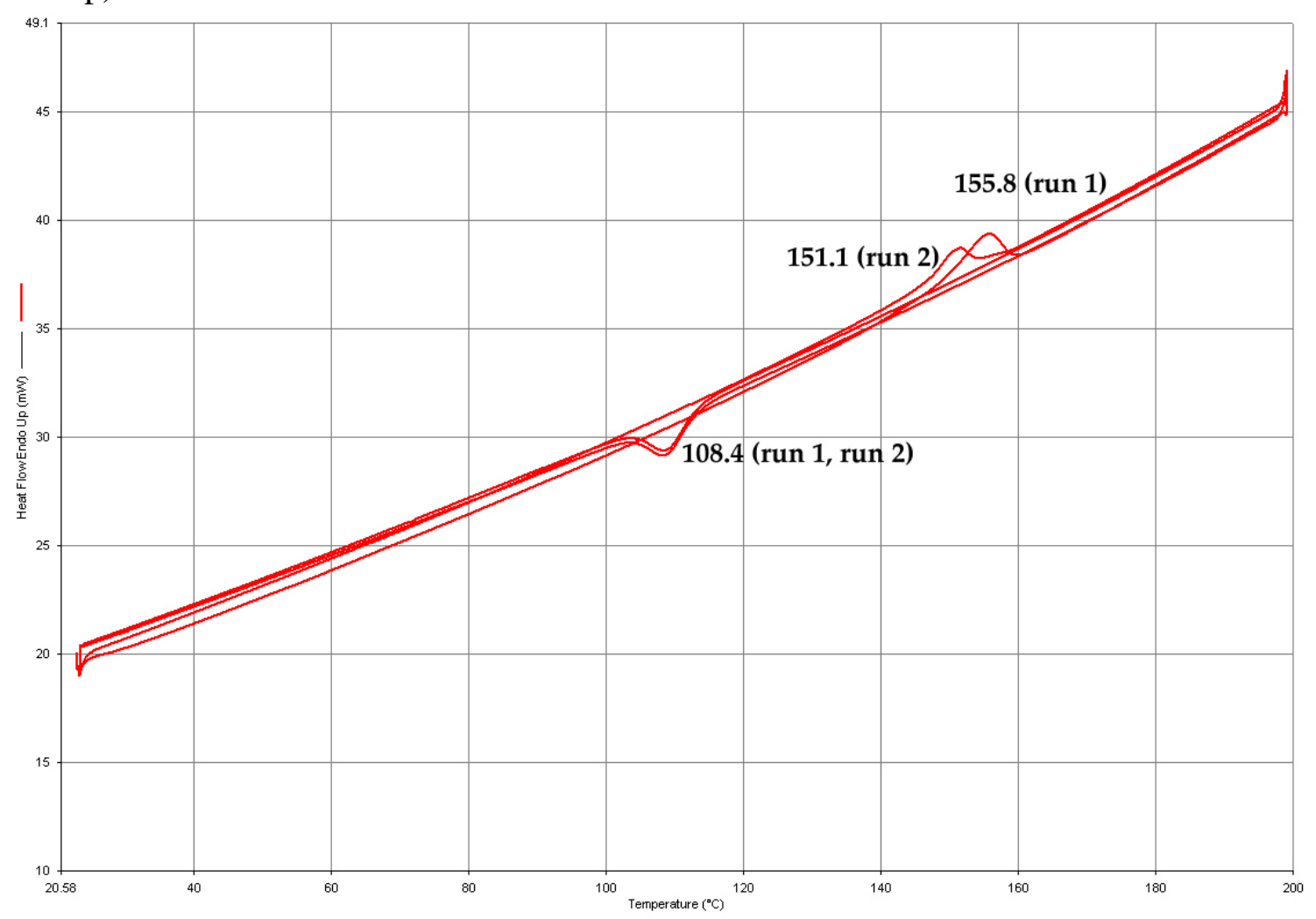

Entry 6: $T_{\mathrm{m}}=$ Amorphous

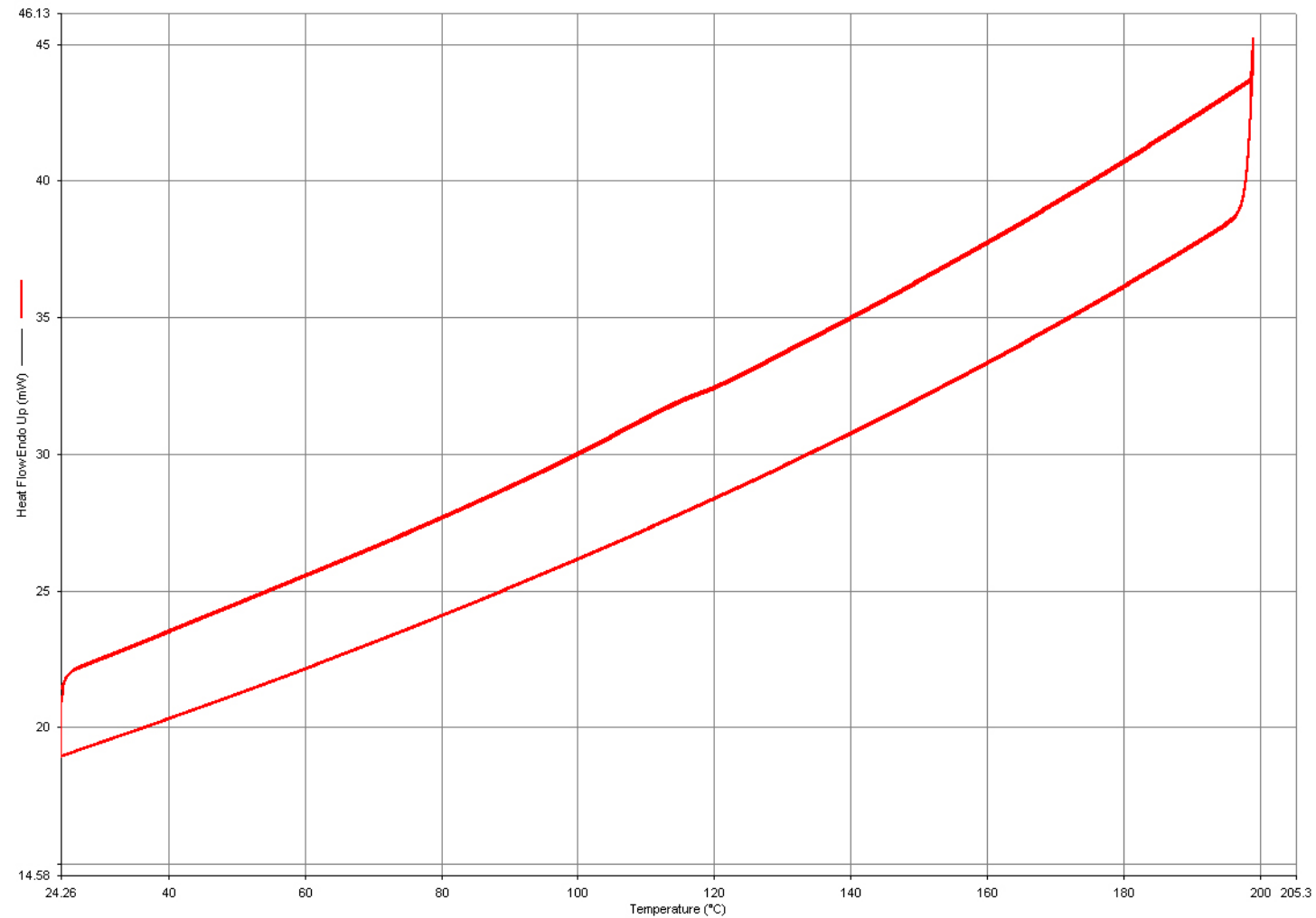


Entry 7: $T_{\mathrm{m}}=165^{\circ} \mathrm{C}$

(endotherm-down)

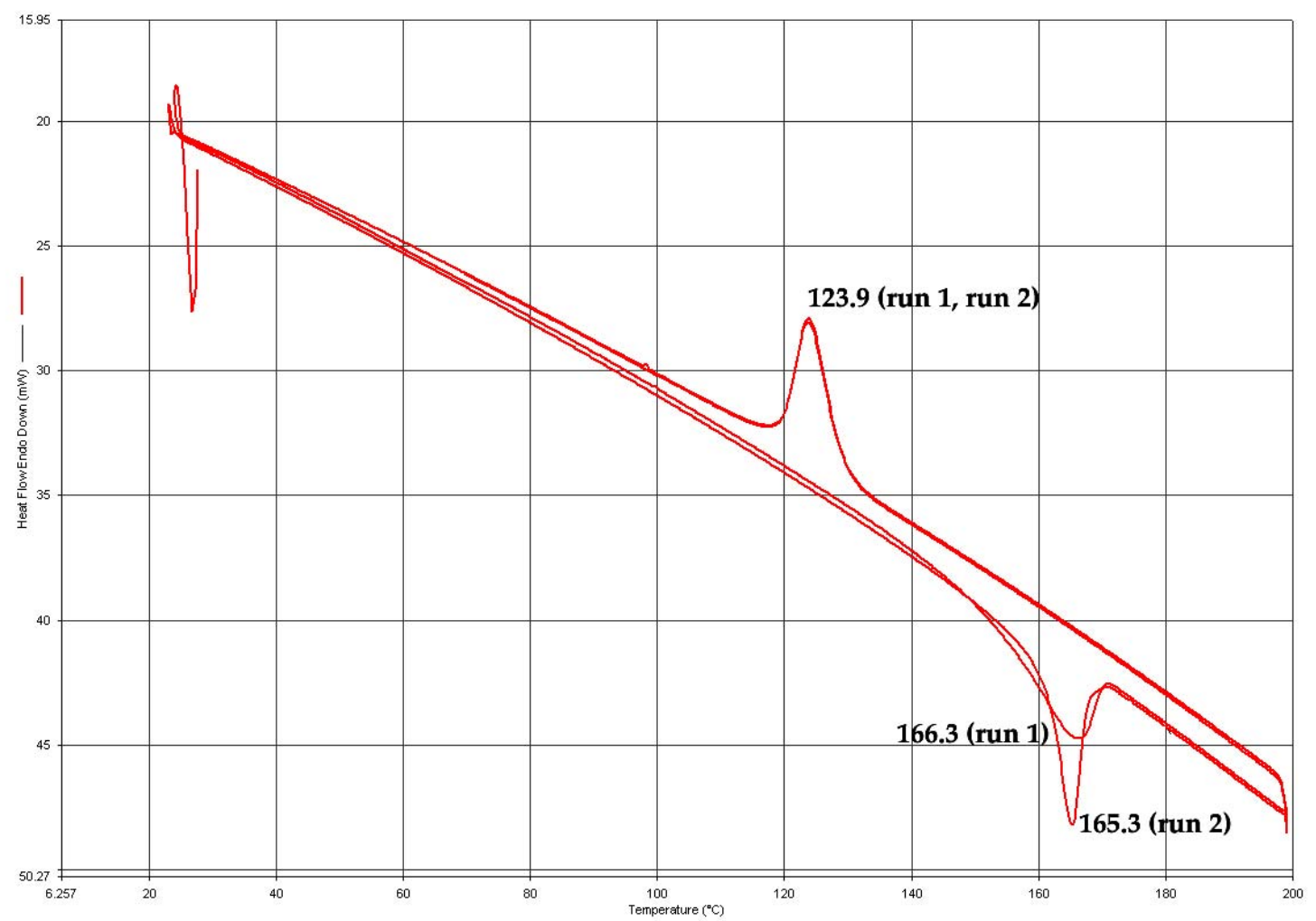

Entry 8: $T_{\mathrm{m}}=164^{\circ} \mathrm{C}$

(endotherm-down)

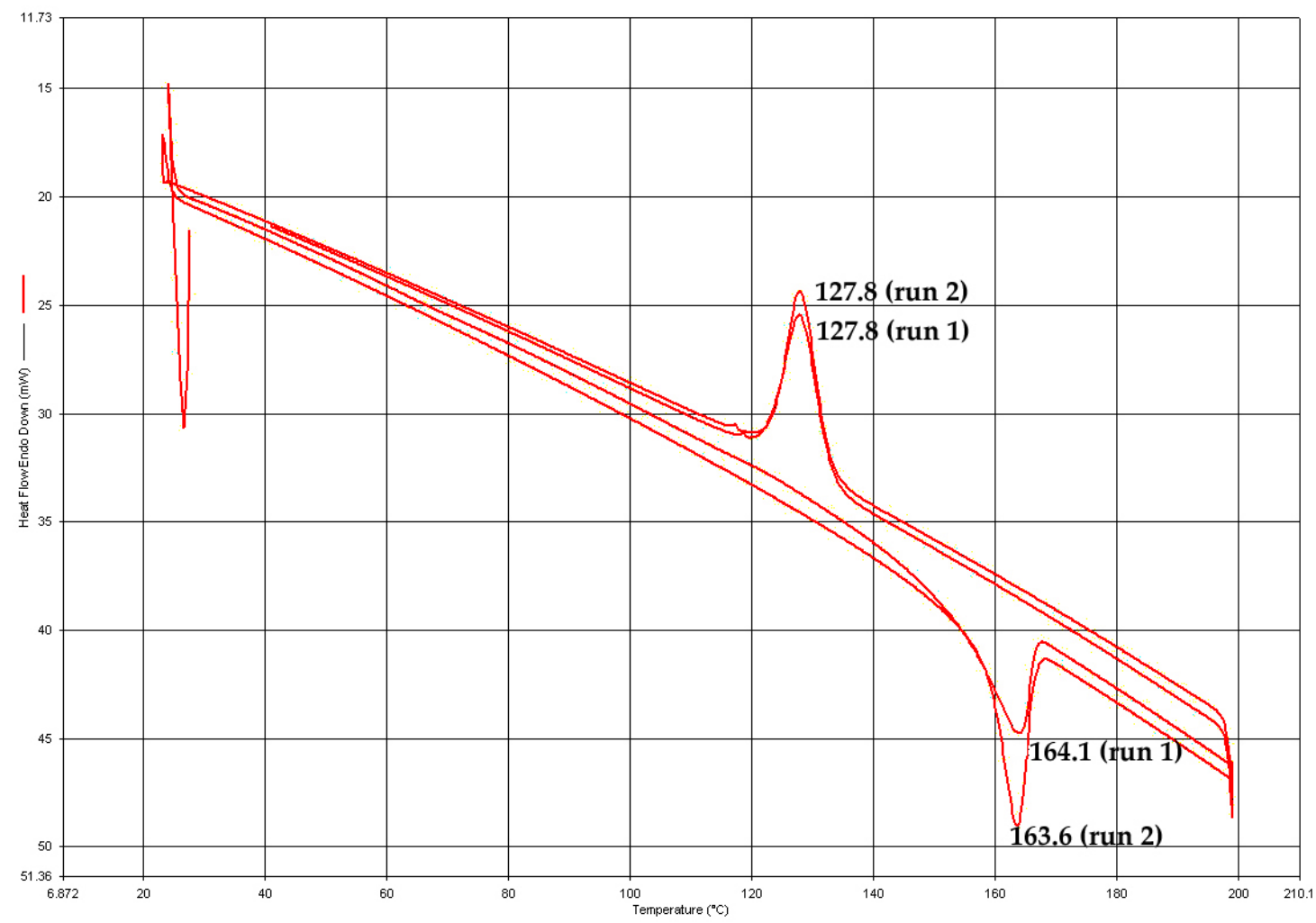


Entry 8: Annealed at $160^{\circ} \mathrm{C}$ for 3 Hours: $T_{\mathrm{m}}=174^{\circ} \mathrm{C}$ (endotherm-up)

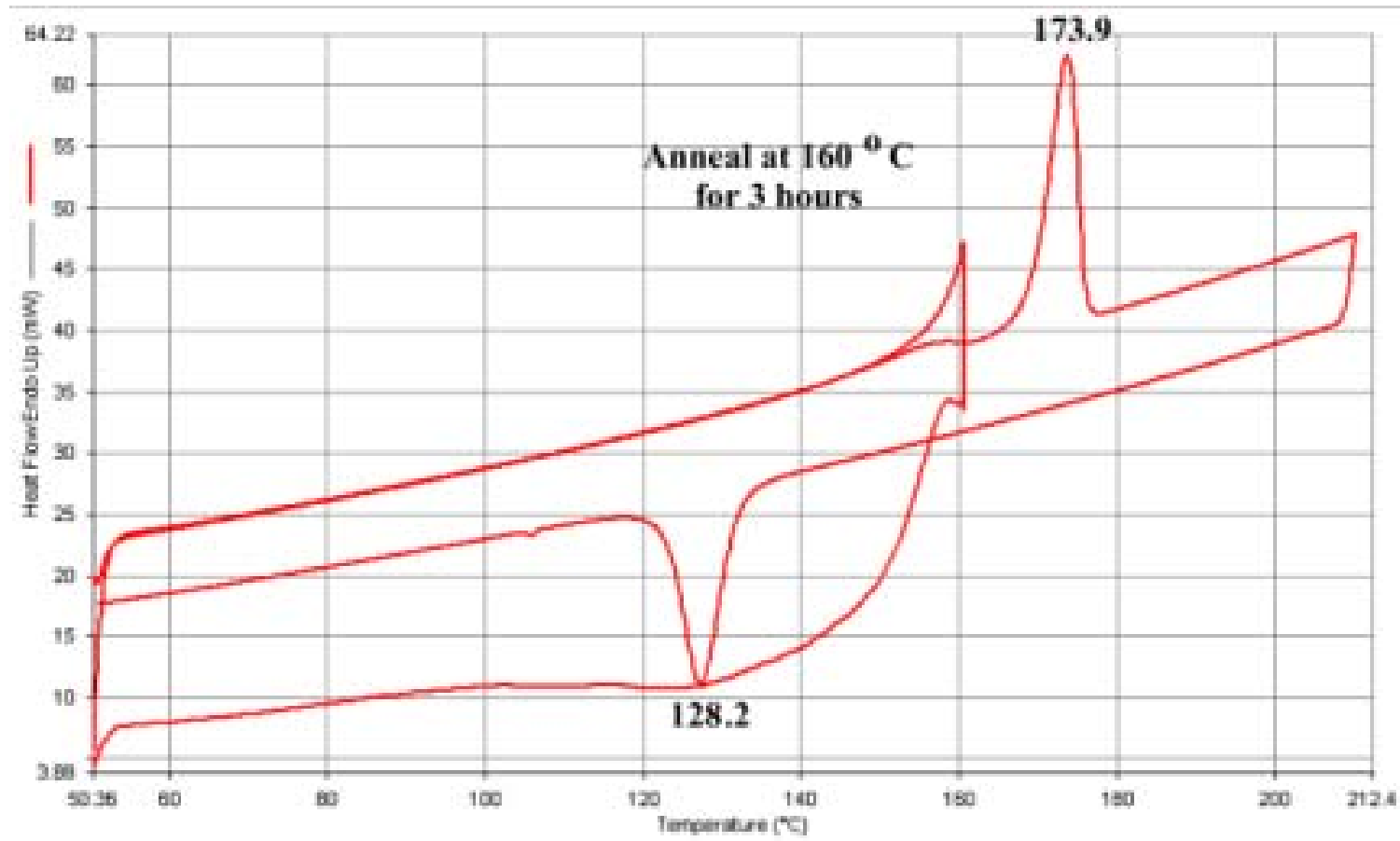

Entry 9: $T_{\mathrm{m}}=157^{\circ} \mathrm{C}$ (endotherm-up)

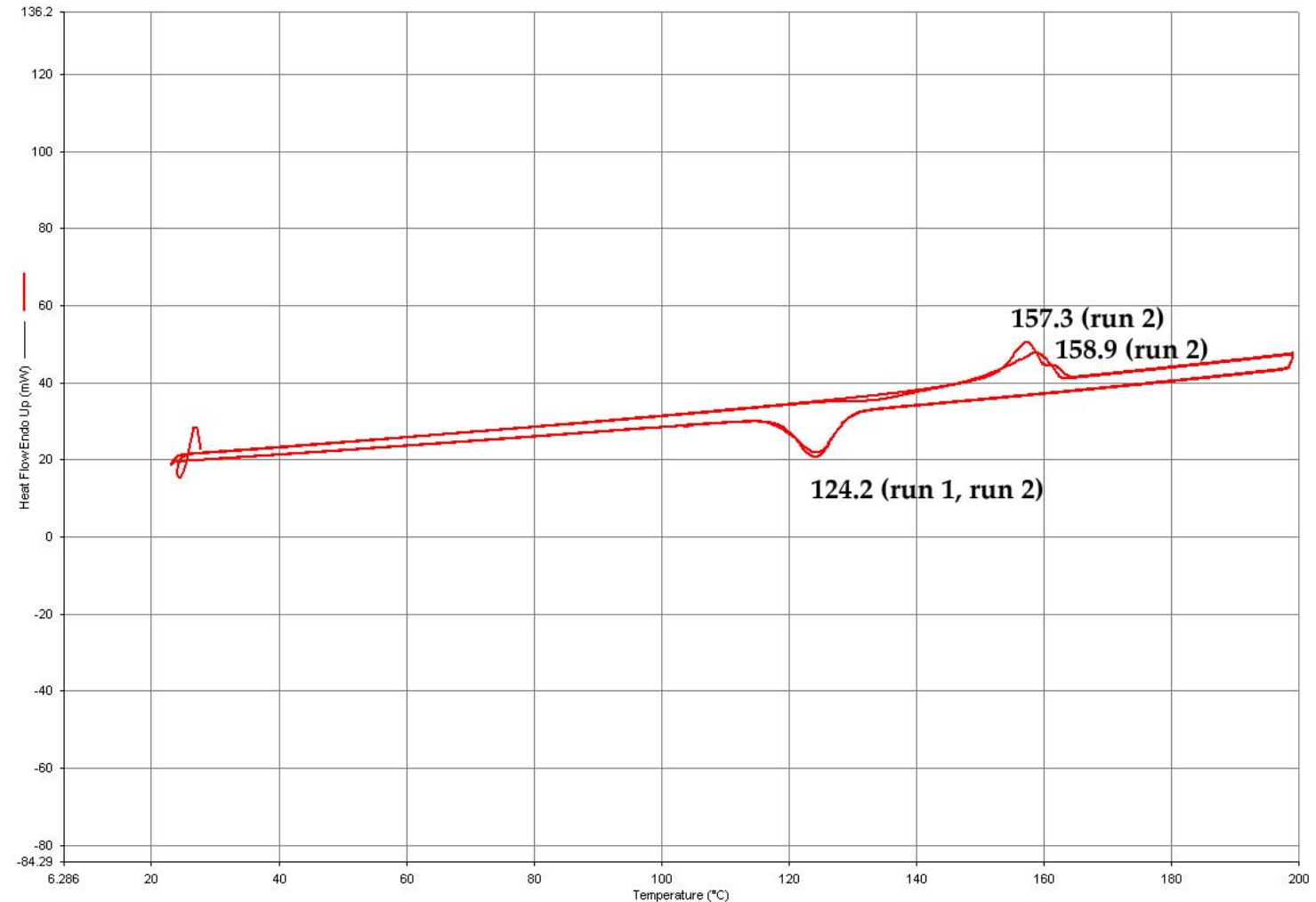


Entry 10: $T_{\mathrm{m}}=128^{\circ} \mathrm{C}$

(endotherm-up)

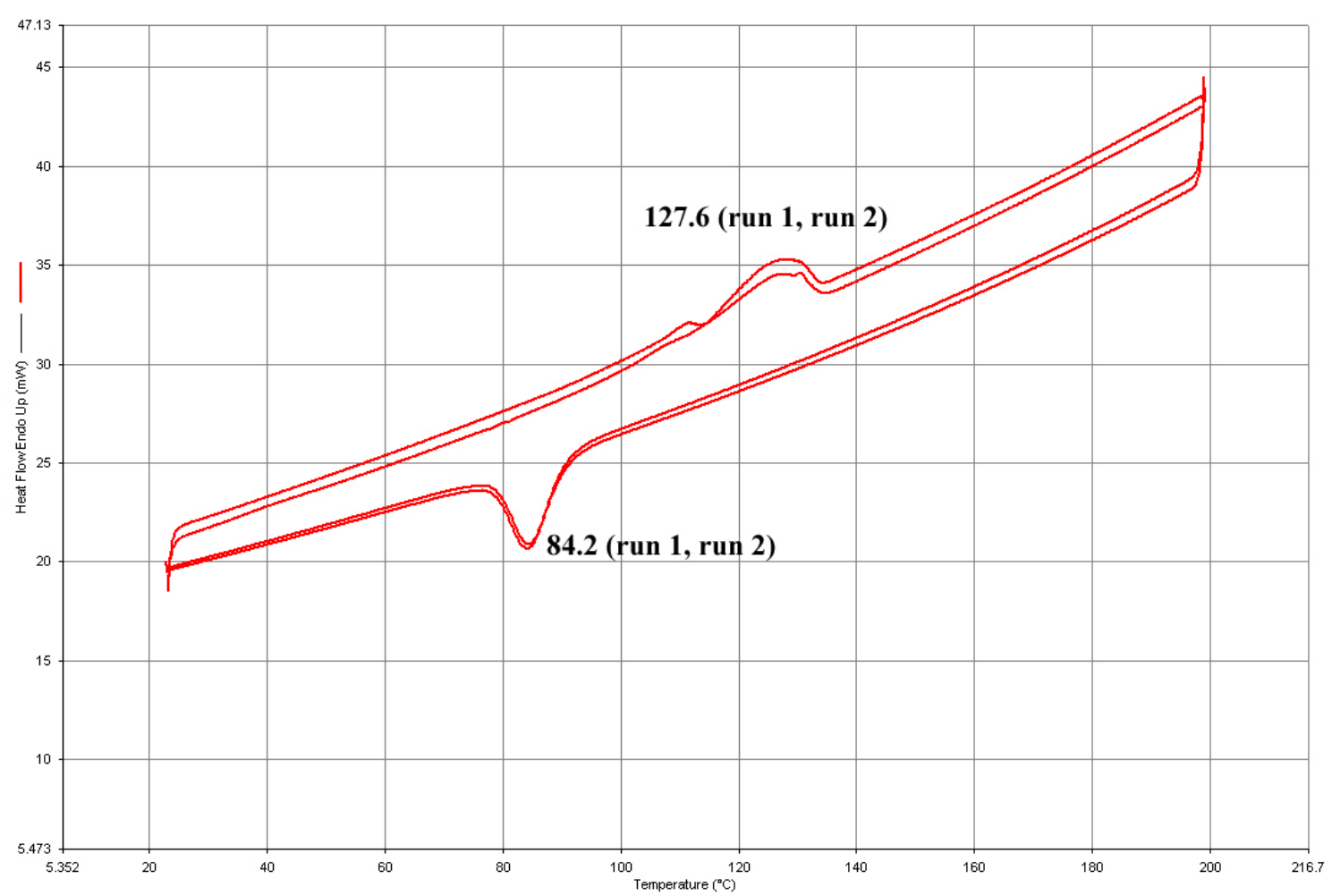


$s$-PP Sample Prepared with $\left[\mathrm{C}_{3} \mathrm{H}_{6}\right]=3.6 \mathrm{M}$ and $T_{\mathrm{p}}=45^{\circ} \mathrm{C}$

MAO-activated propylene polymerization results for $\mathbf{3}^{\mathrm{a}}$

\begin{tabular}{cccccc}
\hline $\begin{array}{c}\text { Catalyst } \\
(\mu \mathrm{mol})\end{array}$ & $\begin{array}{c}T_{\mathrm{p}} \\
\left({ }^{\circ} \mathrm{C}\right)\end{array}$ & $\begin{array}{c}\text { Yield } \\
(\mathrm{g})\end{array}$ & Activity $^{\mathrm{b}}$ & $\begin{array}{c}T_{\mathrm{m}}^{\mathrm{c}} \\
\left({ }^{\circ} \mathrm{C}\right)\end{array}$ & $\begin{array}{c}{[r r r r]} \\
\%^{\mathrm{d}}\end{array}$ \\
\hline $\mathbf{3}(1.66)$ & 45 & 3.52 & 42,400 & 145 & 88 \\
\hline
\end{tabular}

${ }^{a}$ Polymerization conditions: propylene $(10 \mathrm{~mL})$ and toluene $(30 \mathrm{~mL})$; 1000 eq. MAO; 3 min. run. ${ }^{b} \mathrm{~kg} \mathrm{PP} /(\mathrm{mol} \mathrm{M} \cdot \mathrm{h})$. ' Unannealed, determined by DSC. ${ }^{\mathrm{d}}[\mathrm{rrrr}] \%$ determined by ${ }^{13} \mathrm{C}$ NMR.

Full pentad distribution for the polypropylene sample prepared with $\left[\mathrm{C}_{3} \mathrm{H}_{6}\right]=3.6 \mathrm{M}$ and $T_{\mathrm{p}}=45^{\circ} \mathrm{C}$

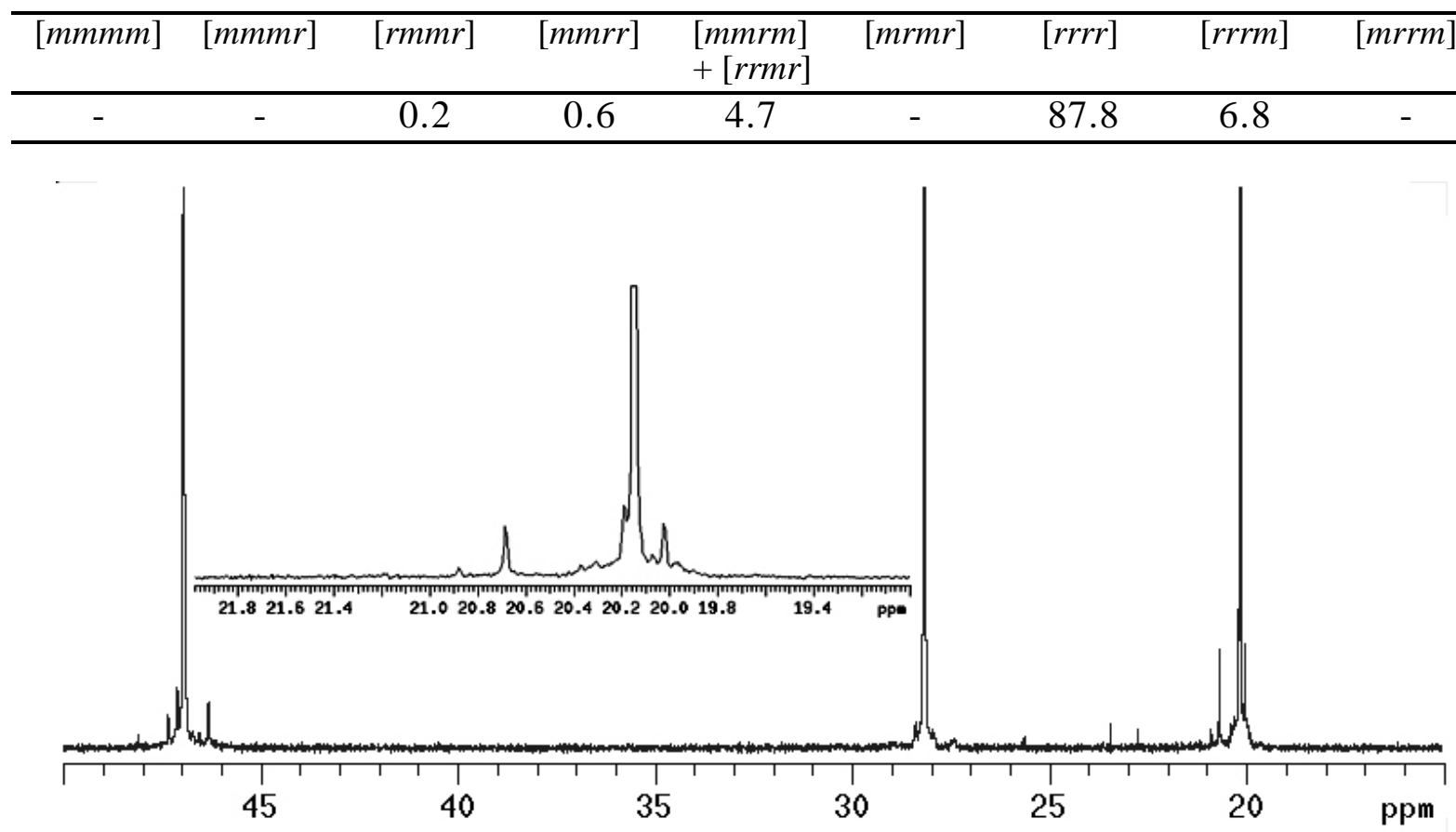

$T_{\mathrm{m}}=\mathbf{1 4 5}^{\circ} \mathrm{C}$ (endotherm-up)

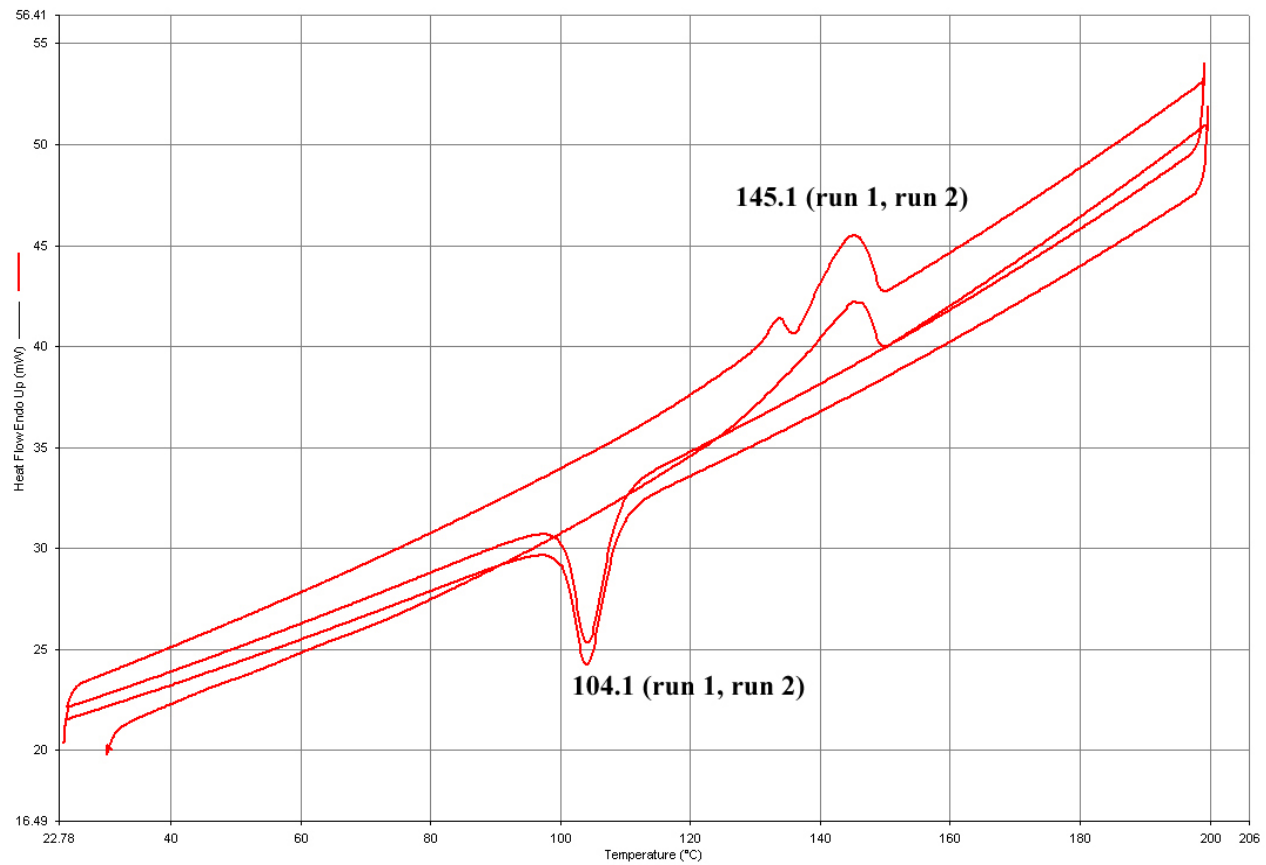




\section{${ }^{13}$ C NMR Spectra of $s$-P4M1P Samples Reported in Table 2}

(Insets are enlargements of the side-chain methylene region, the 3-carbon of the monomer)

Table 2. MAO-activated 4-methyl-1-pentene polymerization results for $\mathbf{1 - 3}$. $^{\text {a }}$

\begin{tabular}{ccccrcc}
\hline Entry & $\begin{array}{c}\text { Catalyst } \\
(\mu \mathrm{mol})\end{array}$ & $\begin{array}{c}T_{\mathrm{p}} \\
\left({ }^{\circ} \mathrm{C}\right)\end{array}$ & $\begin{array}{c}\text { Yield } \\
(\mathrm{g})\end{array}$ & Activity $^{\mathrm{b}}$ & $\begin{array}{c}T_{\mathrm{m}}^{\mathrm{c}} \\
\left({ }^{\circ} \mathrm{C}\right)\end{array}$ & $\begin{array}{c}{[\mathrm{rrrr}]} \\
\%^{\mathrm{d}}\end{array}$ \\
\hline 15 & $\mathbf{1}(1.62)$ & 0 & 0.61 & 500 & 210 & 94 \\
16 & $\mathbf{1}(1.62)$ & 25 & 0.17 & 420 & 206 & 93 \\
17 & $\mathbf{2}(1.53)$ & 0 & 0.00 & 0 & - & - \\
18 & $\mathbf{2}(1.53)$ & 25 & 0.03 & 80 & am. & - \\
19 & $\mathbf{3}(1.66)$ & 0 & 0.47 & 380 & 215 & 97 \\
20 & $\mathbf{3}(1.66)$ & 25 & 2.14 & 5,160 & 202 & 89 \\
\hline
\end{tabular}

aPolymerization conditions: neat 4-methyl-1-pentene (25 mL); 1000 eq. MAO; 45 min. runs at $T_{\mathrm{p}}=0^{\circ} \mathrm{C} ; 15 \mathrm{~min}$. runs at $T_{\mathrm{p}}=25^{\circ} \mathrm{C}$. ${ }^{\mathrm{b}} \mathrm{kg} \mathrm{P} 4 \mathrm{M} 1 \mathrm{P} /(\mathrm{mol} \mathrm{M} \cdot \mathrm{h})$. ${ }^{c}$ Unannealed, determined by DSC; am. = amorphous. ${ }^{\mathrm{d}}[\mathrm{rrrr}] \%$ determined by ${ }^{13} \mathrm{C}$ NMR by integration of the peaks in the side-chain methylene region.

Entry 15: $[r r r r]=94 \%$

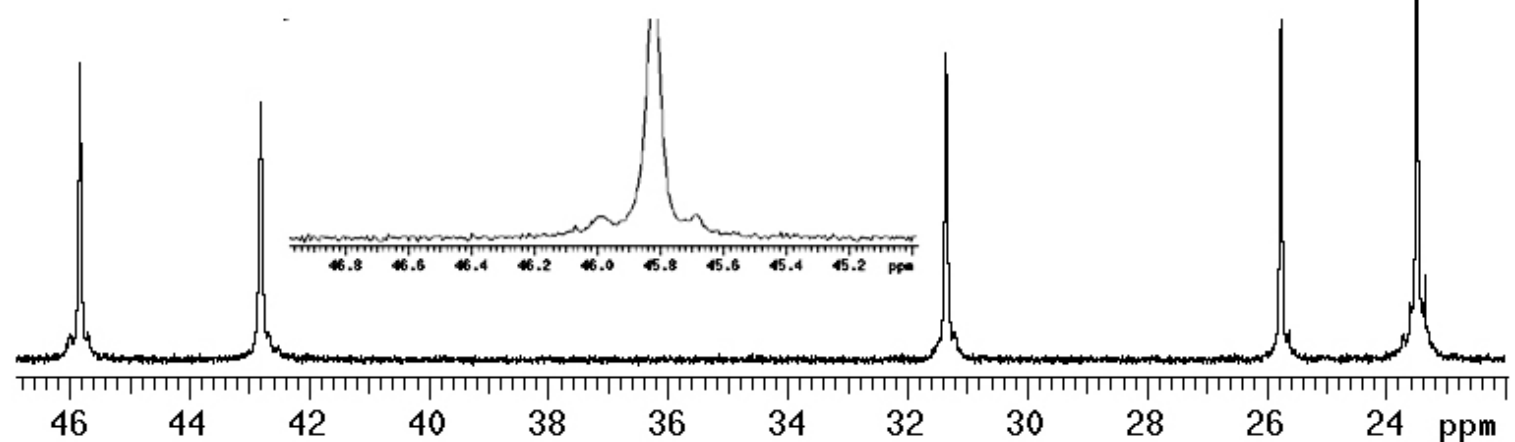

Entry 16: $[r r r r]=93 \%$

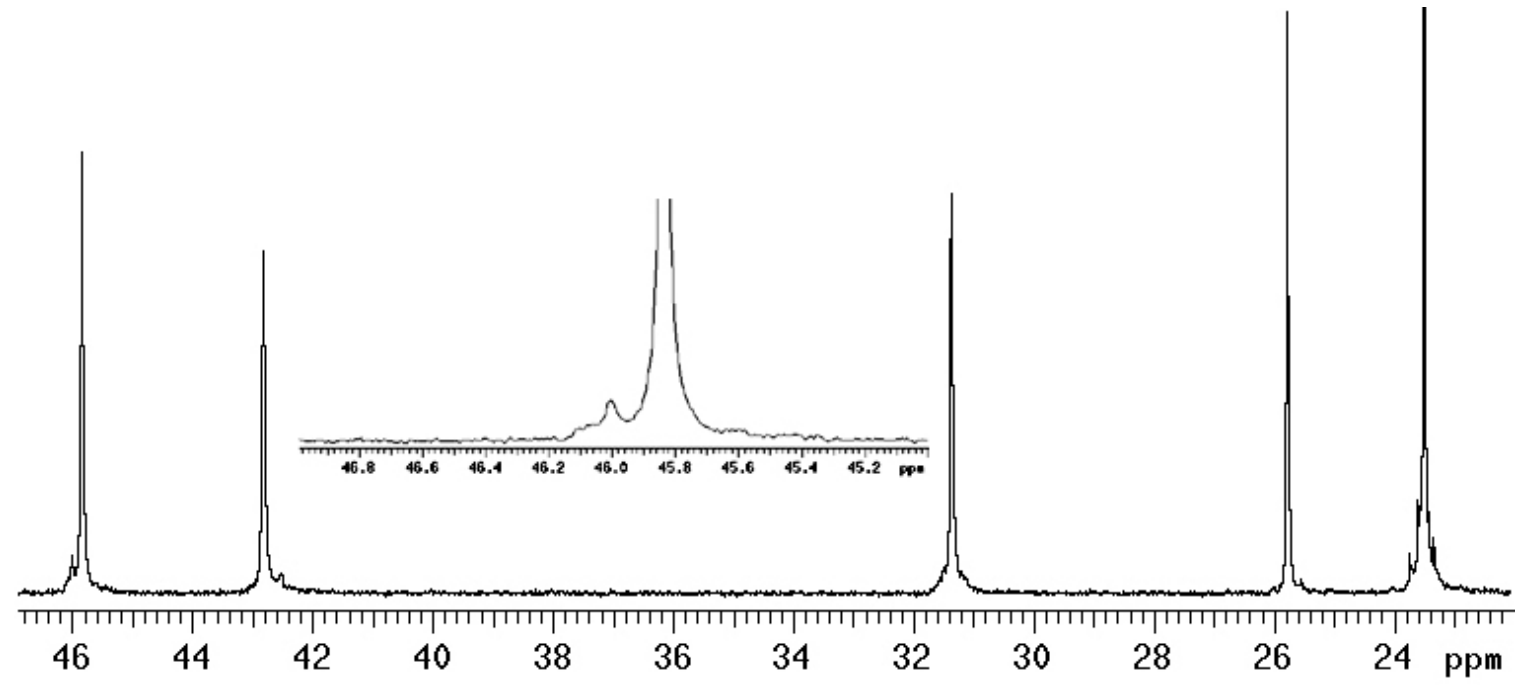


Entry 17: No polymer obtained

Entry 18: Amorphous

Entry 19: $[r r r r]=97 \%$

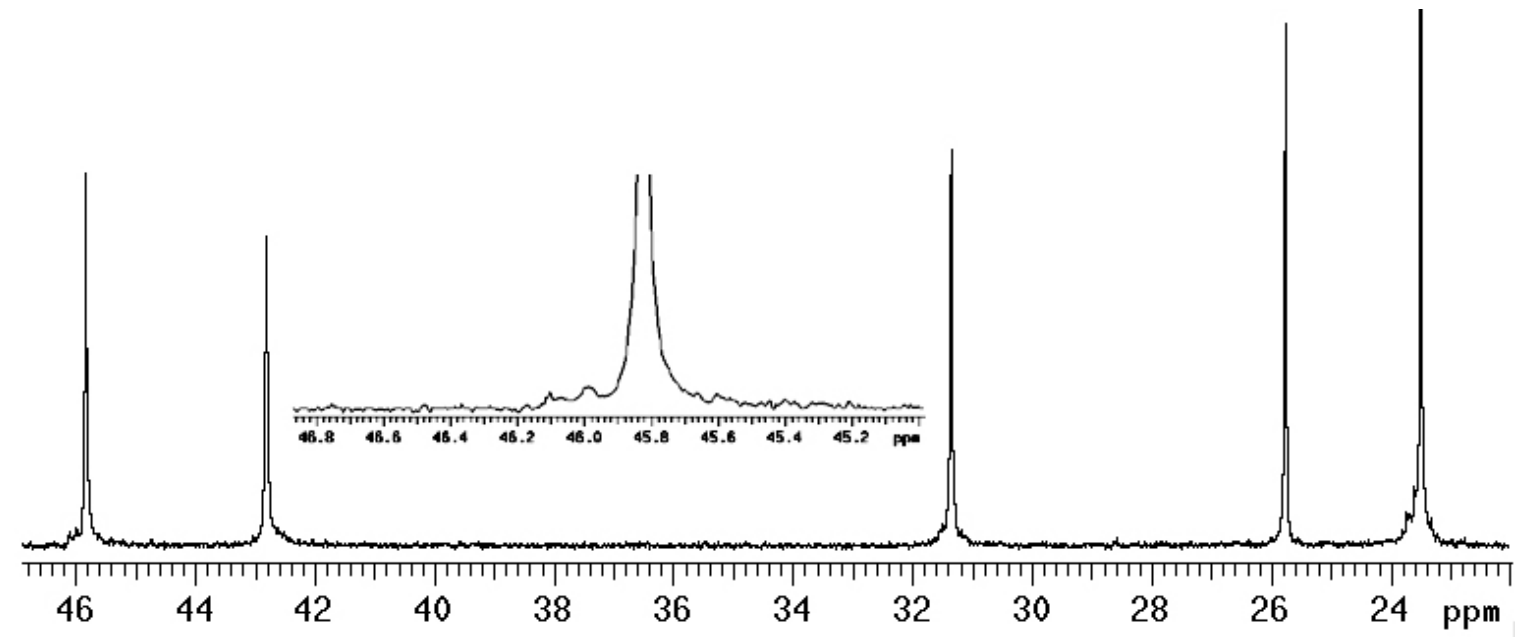

Entry 20: $[r r r r]=89 \%$

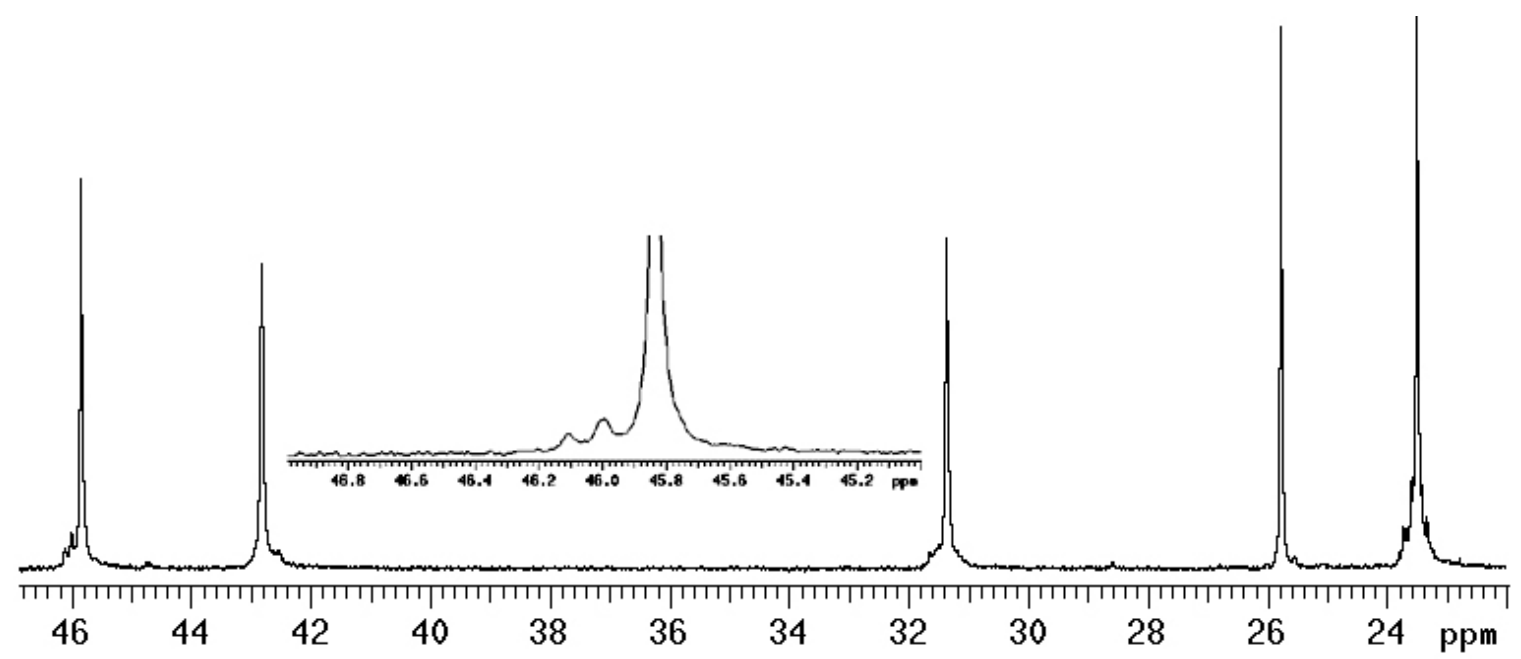


DSC Thermograms of $s$-P4M1P Samples Reported in Table 2

Entry 15: $T_{\mathrm{m}}=210^{\circ} \mathrm{C}$

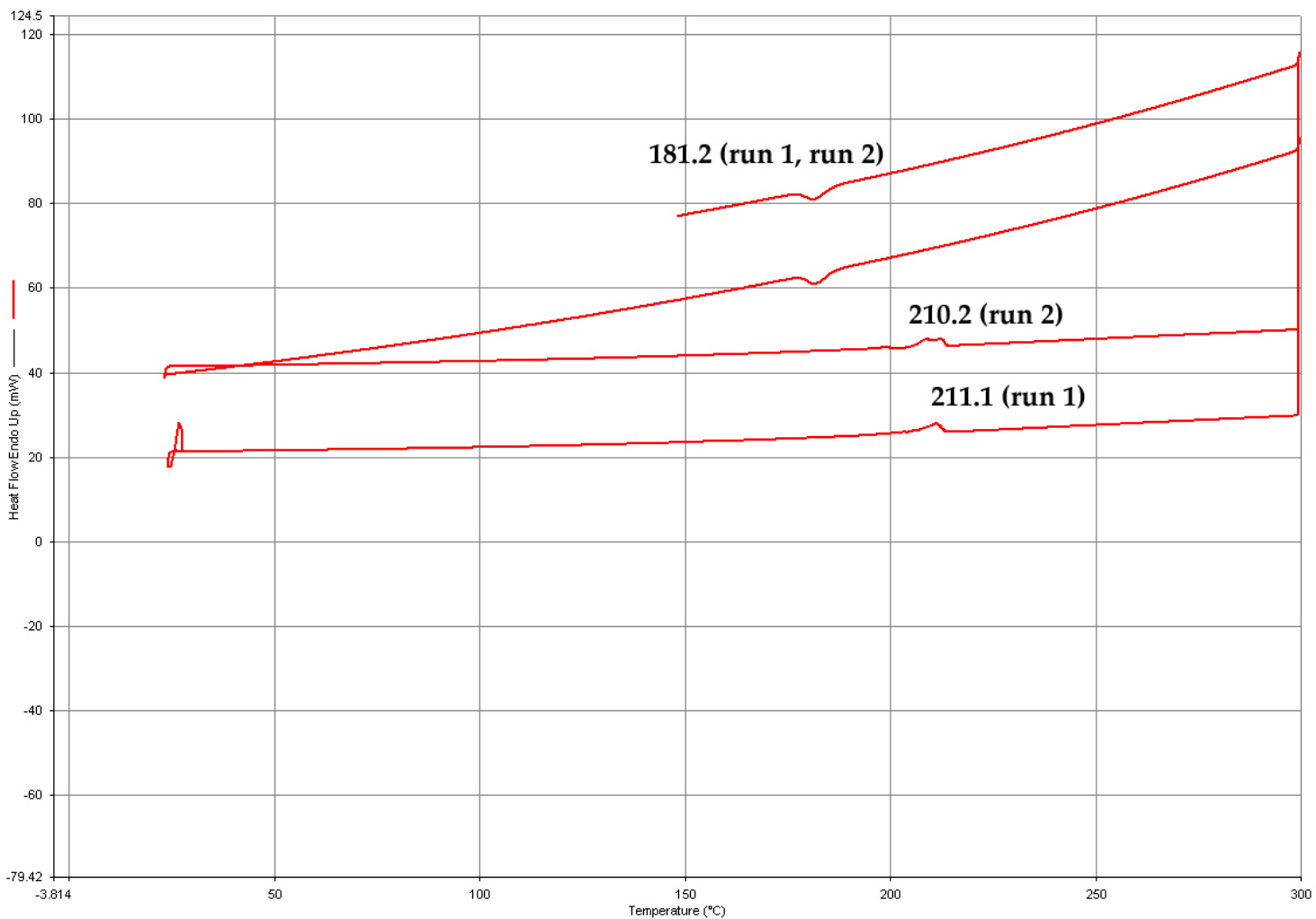

Entry 16: $T_{\mathrm{m}}=206^{\circ} \mathrm{C}$

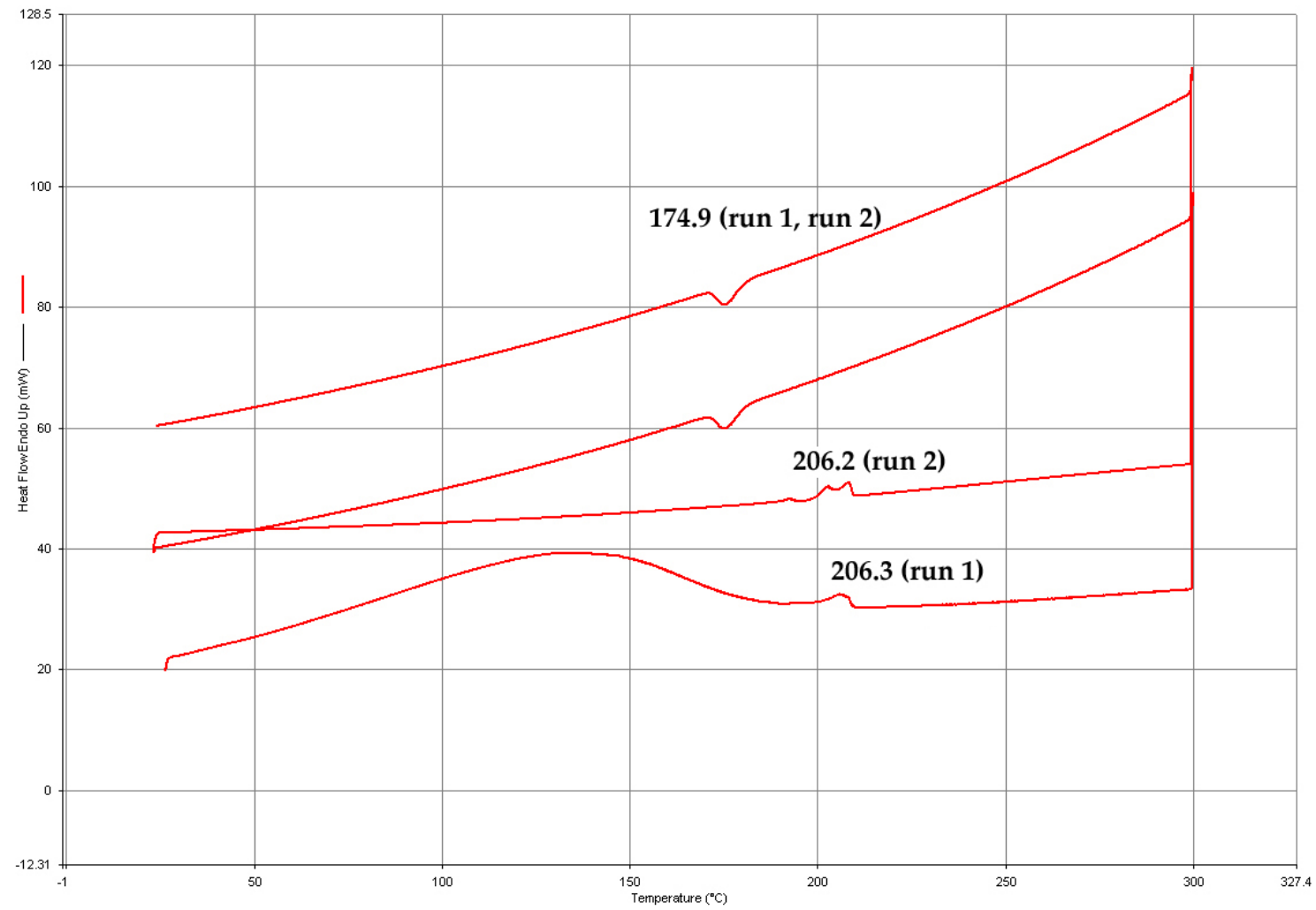


Entry 17: No polymer obtained

Entry 18: Amorphous

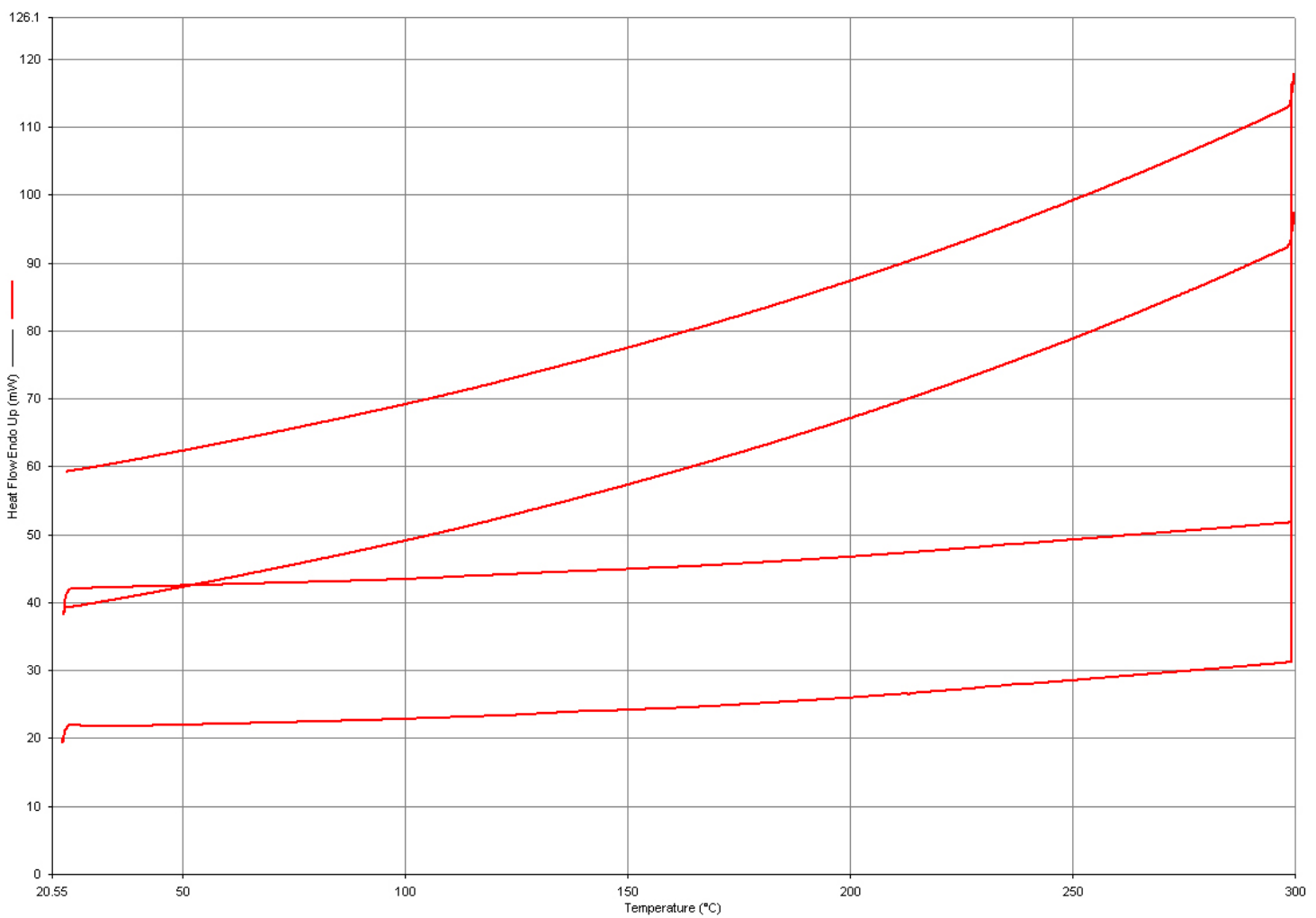

Entry 19: $T_{\mathrm{m}}=215^{\circ} \mathrm{C}$

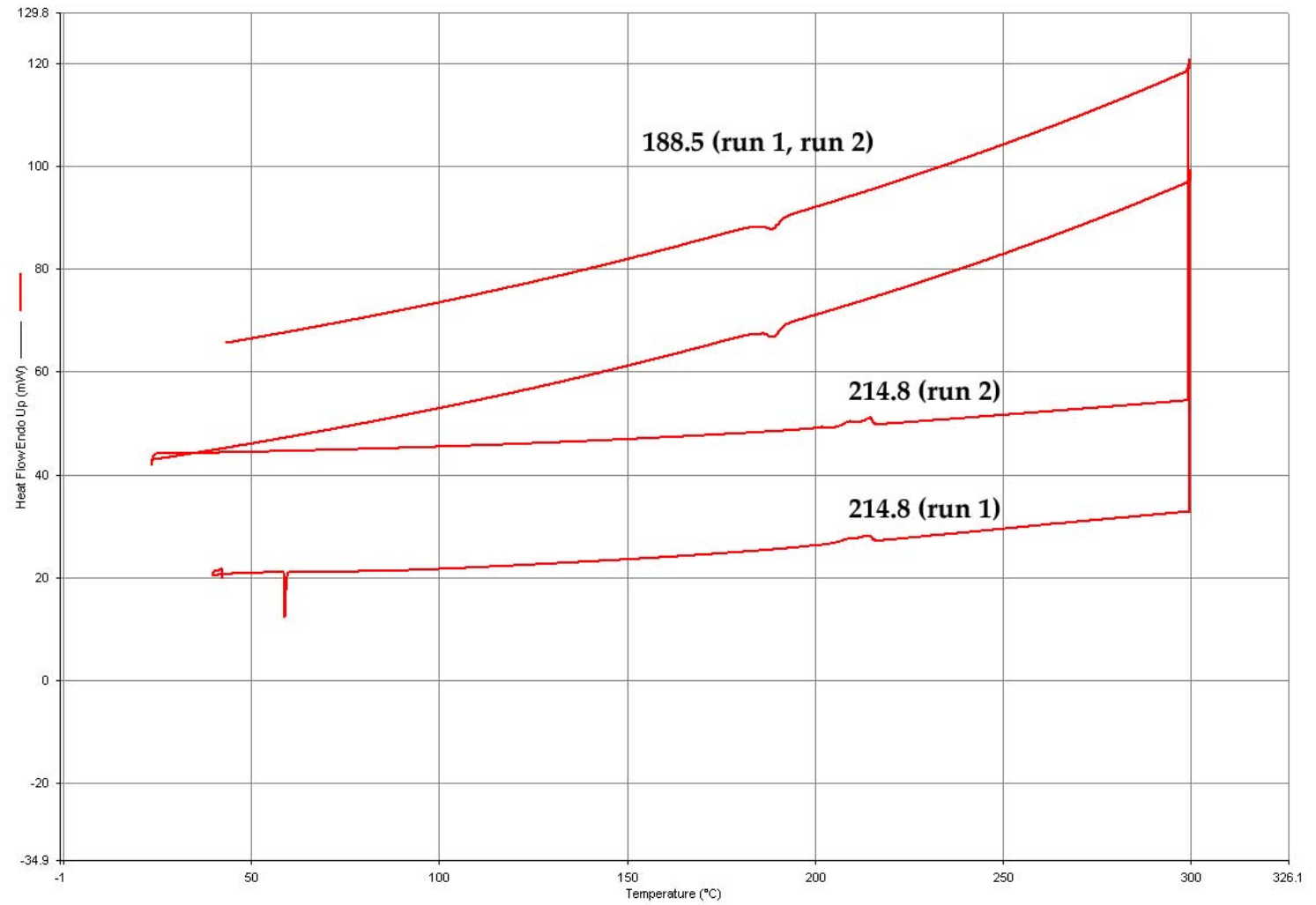


Entry 20: $T_{\mathrm{m}}=202^{\circ} \mathrm{C}$

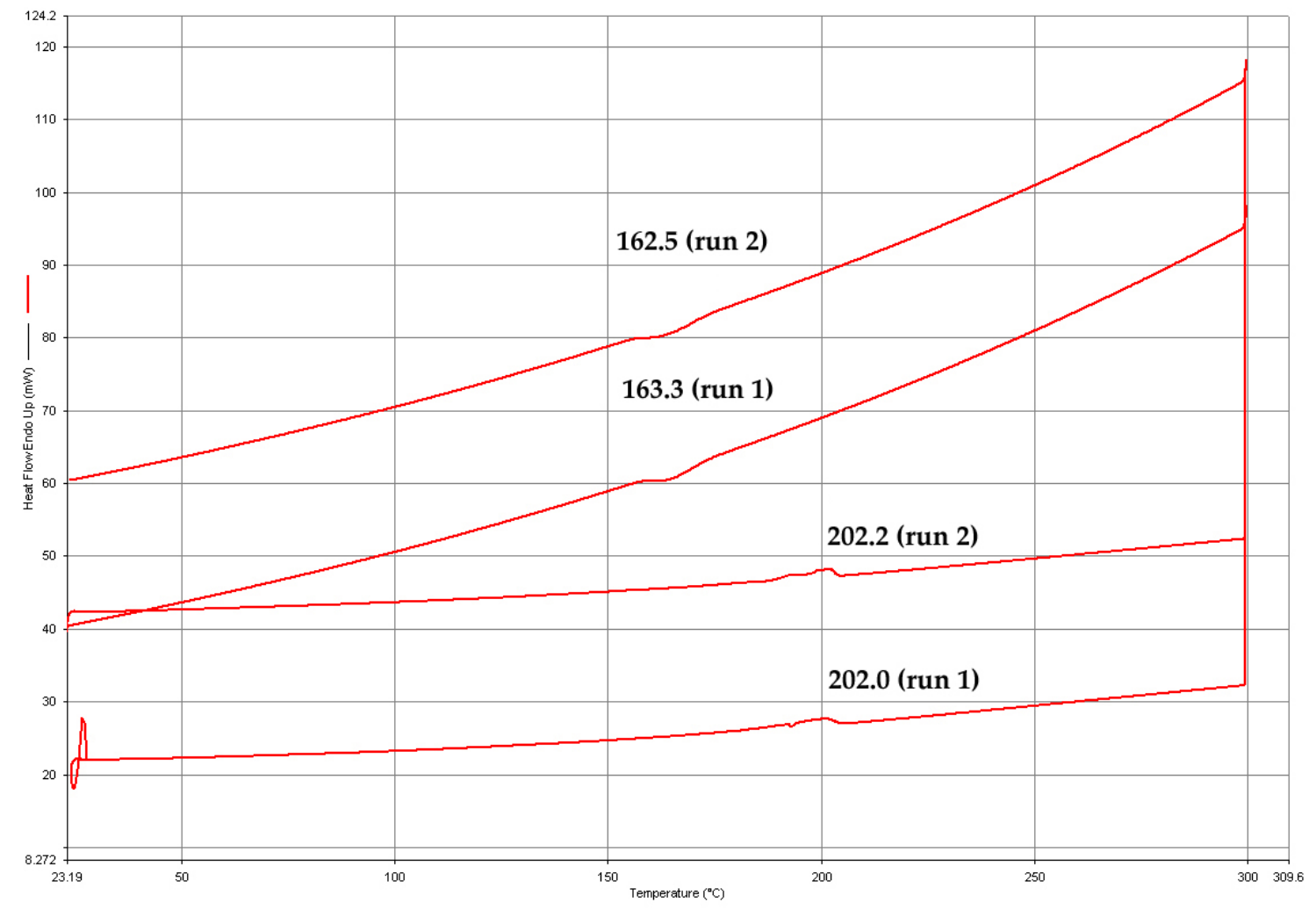




\section{Gel Permeation Chromatography (GPC) Data}

Entry 1: Catalyst 1, $T_{\mathrm{p}}=0^{\circ} \mathrm{C}$
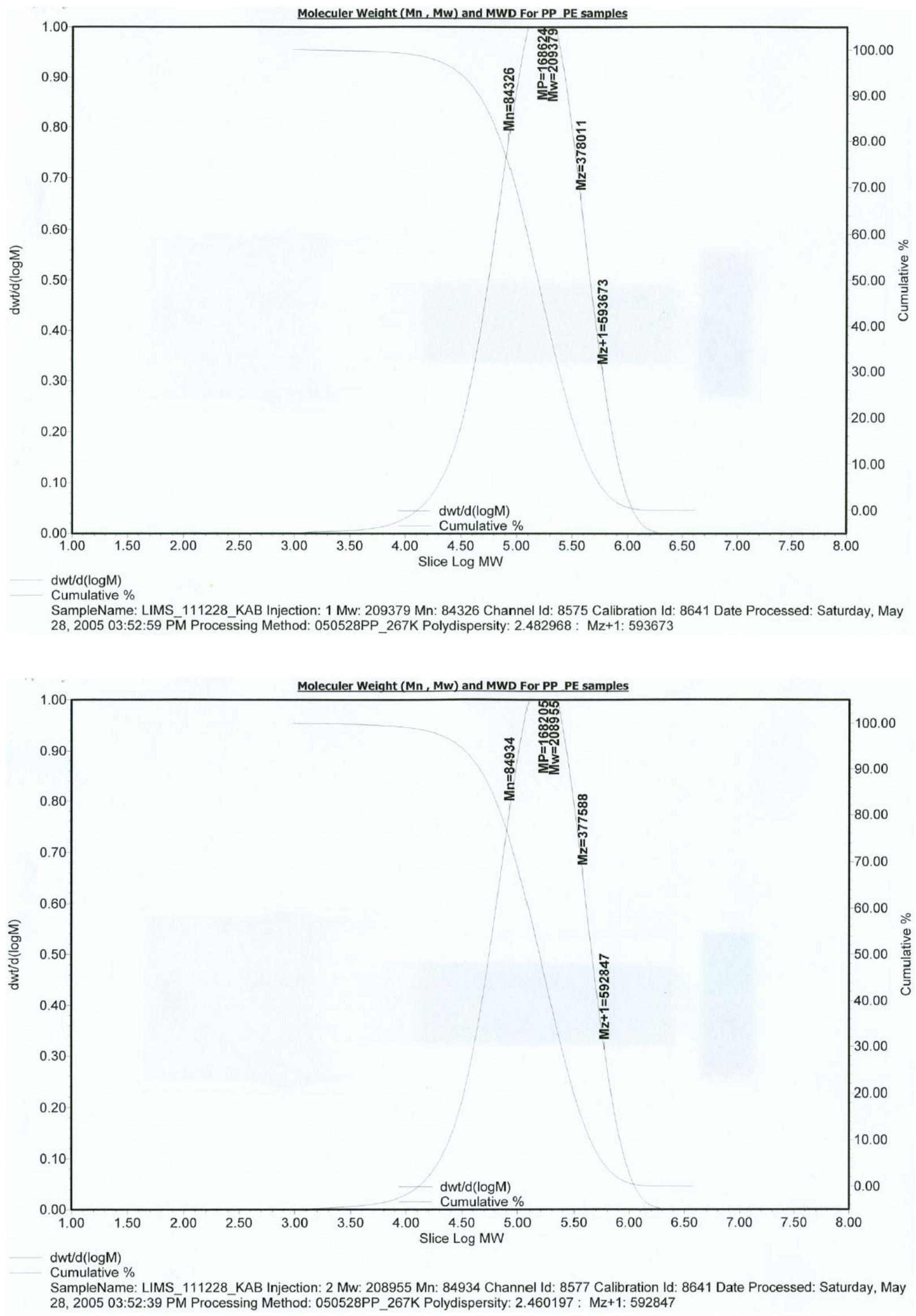
Entry 2: Catalyst $1, T_{\mathrm{p}}=25^{\circ} \mathrm{C}$
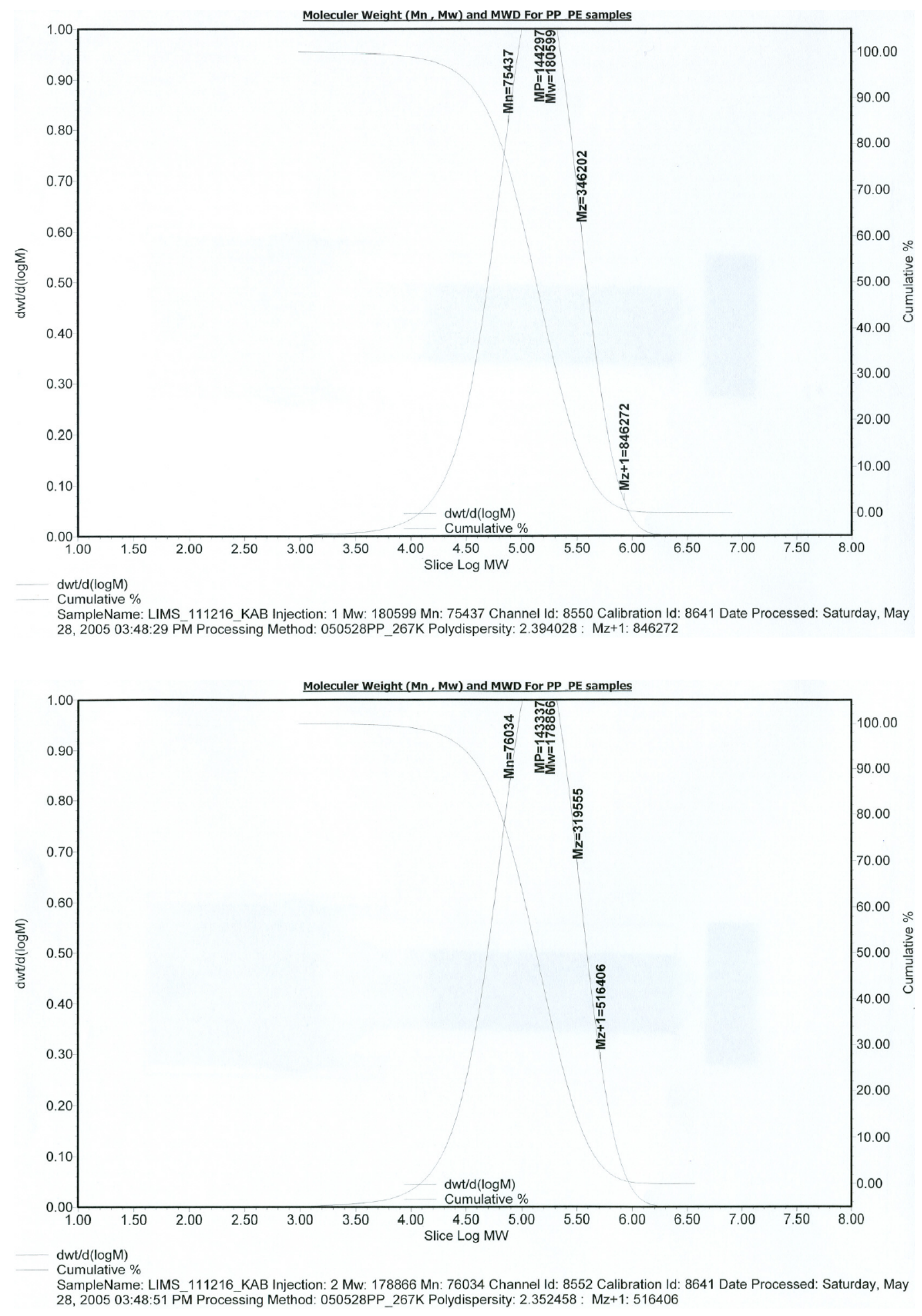
Entry 3: Catalyst 1, $T_{\mathrm{p}}=90^{\circ} \mathrm{C}$
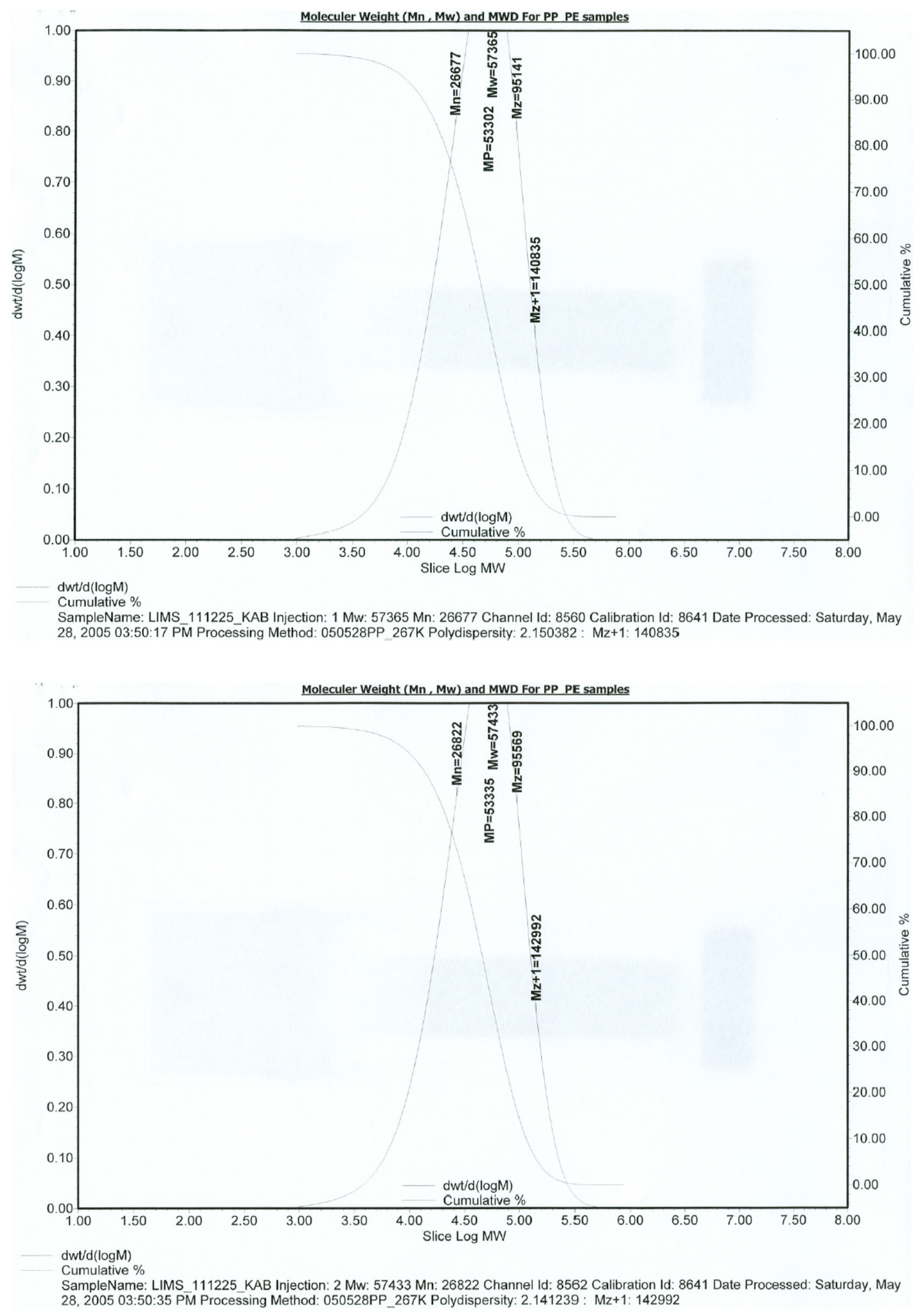
Entry 4: Catalyst 2, $T_{\mathrm{p}}=0^{\circ} \mathrm{C}$
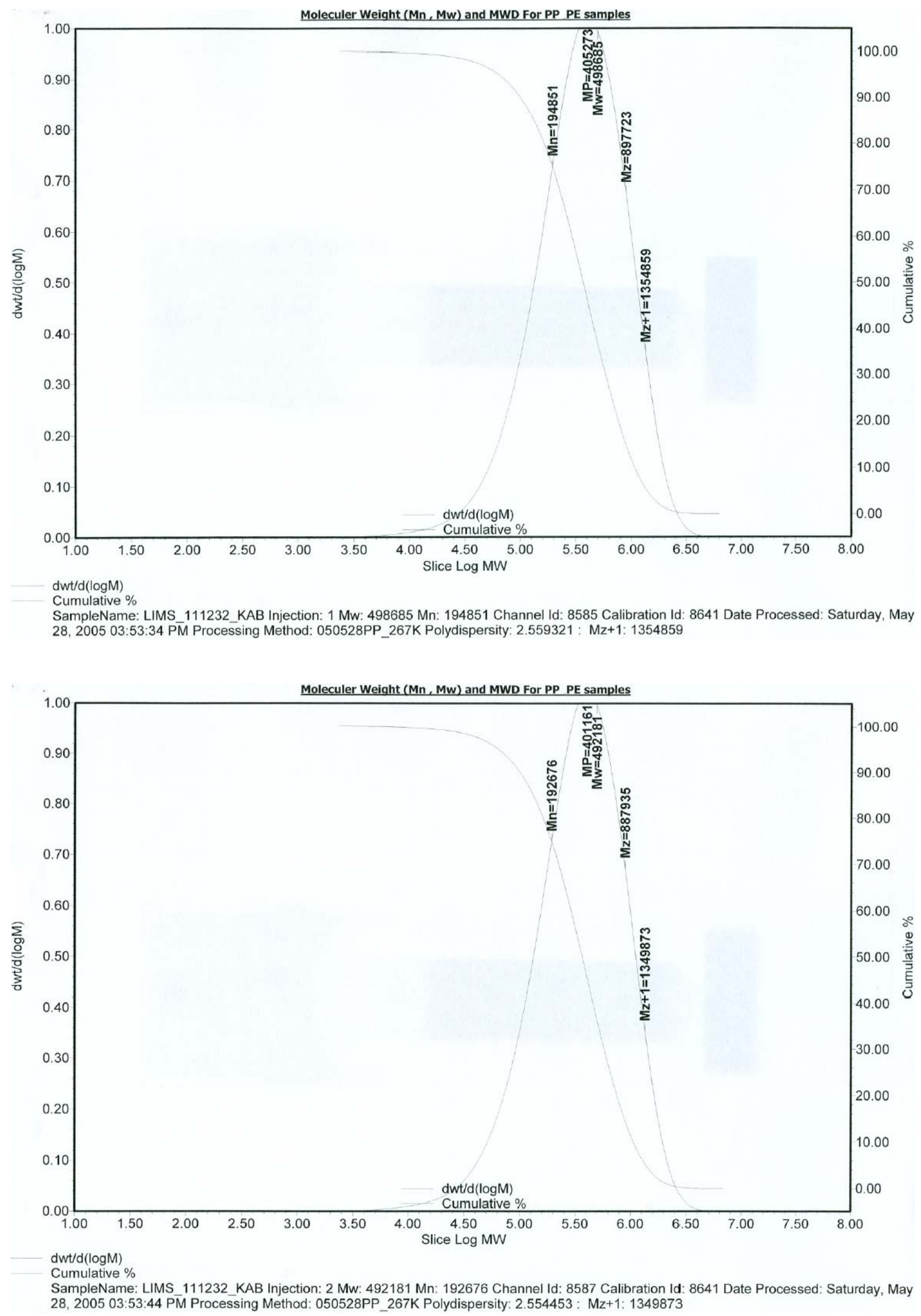
Entry 5: Catalyst 2, $T_{\mathrm{p}}=25^{\circ} \mathrm{C}$
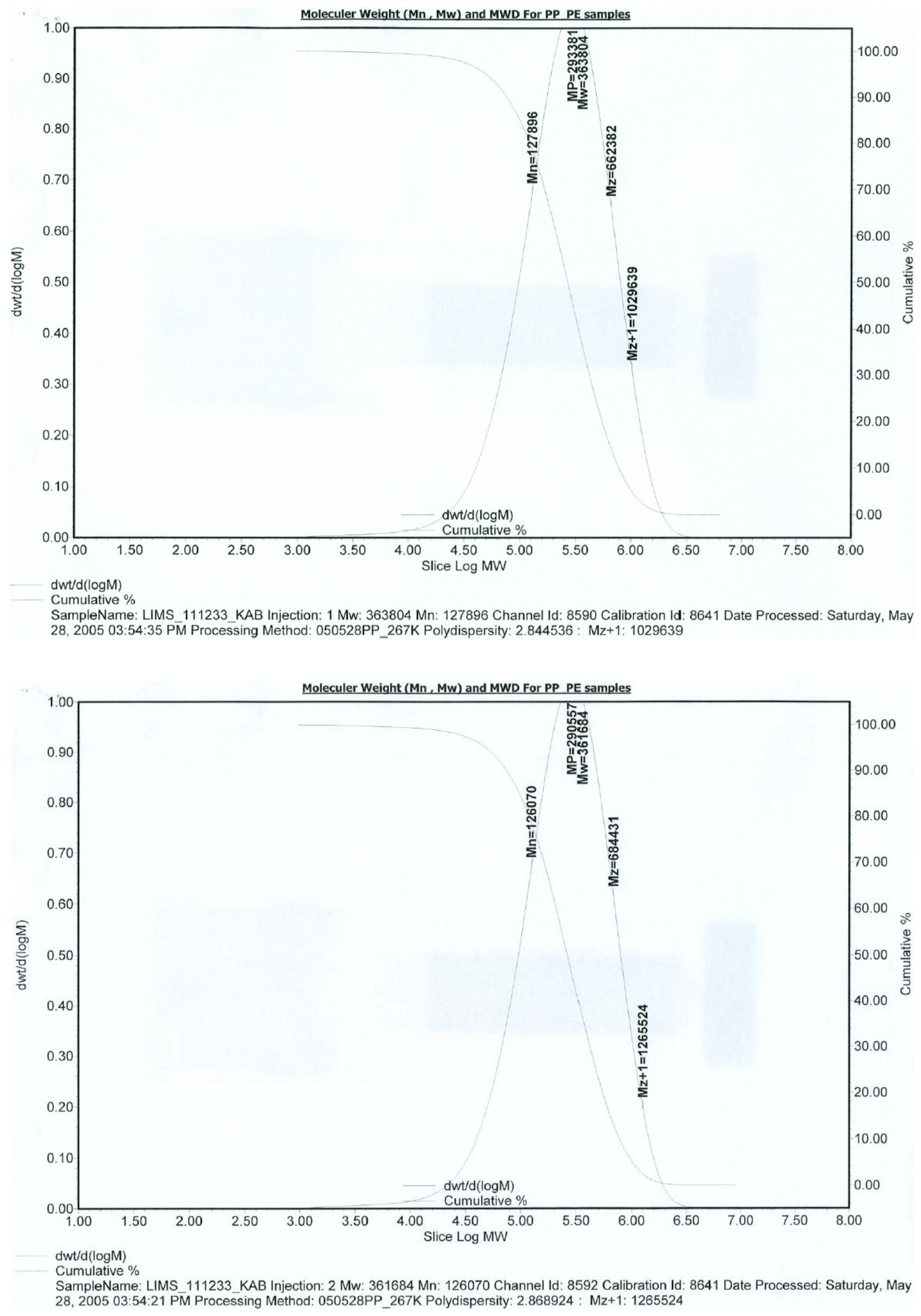
Entry 6: Catalyst 2, $T_{\mathrm{p}}=90^{\circ} \mathrm{C}$
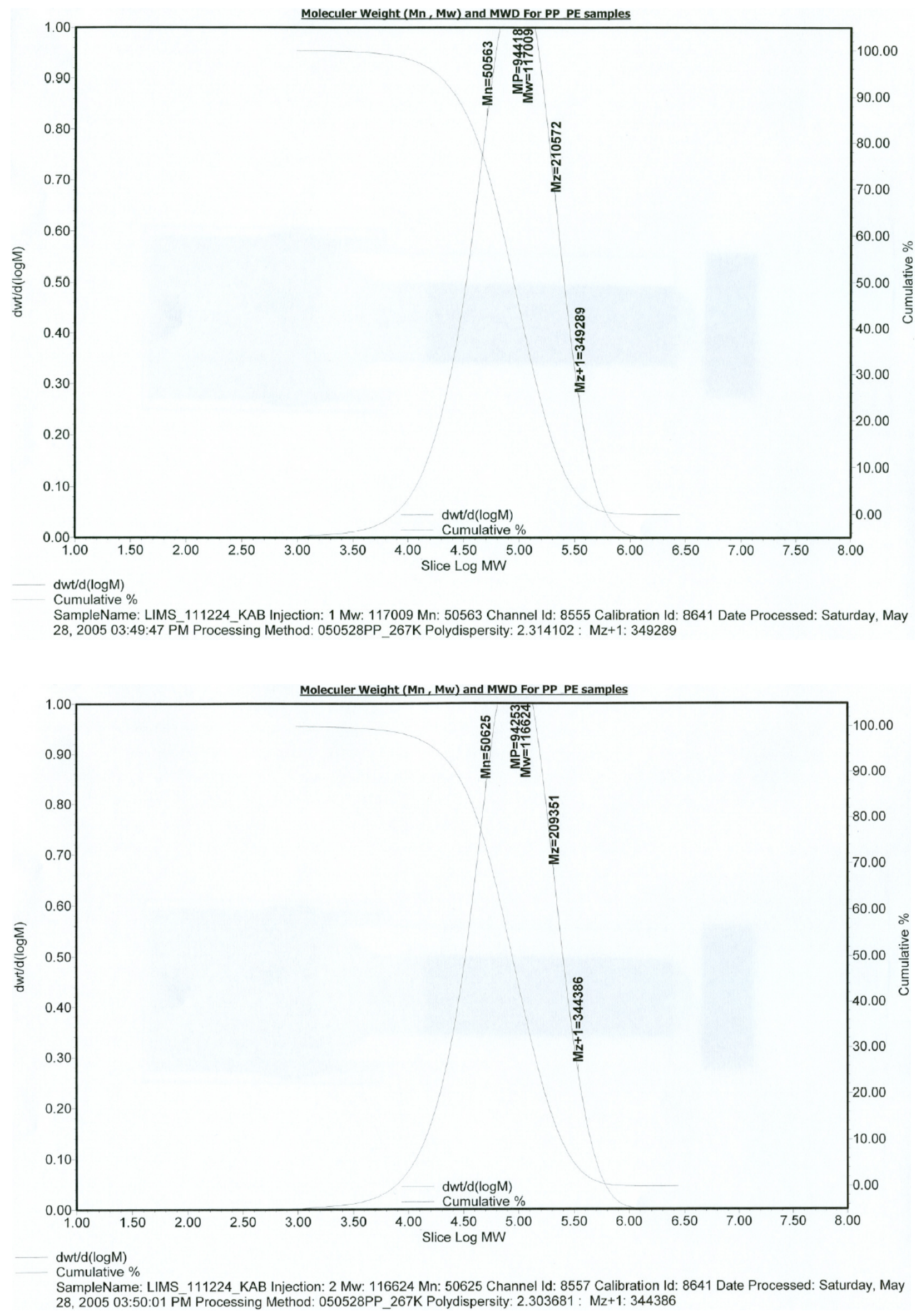
Entry 7: Catalyst 3, $T_{\mathrm{p}}=-15^{\circ} \mathrm{C}$
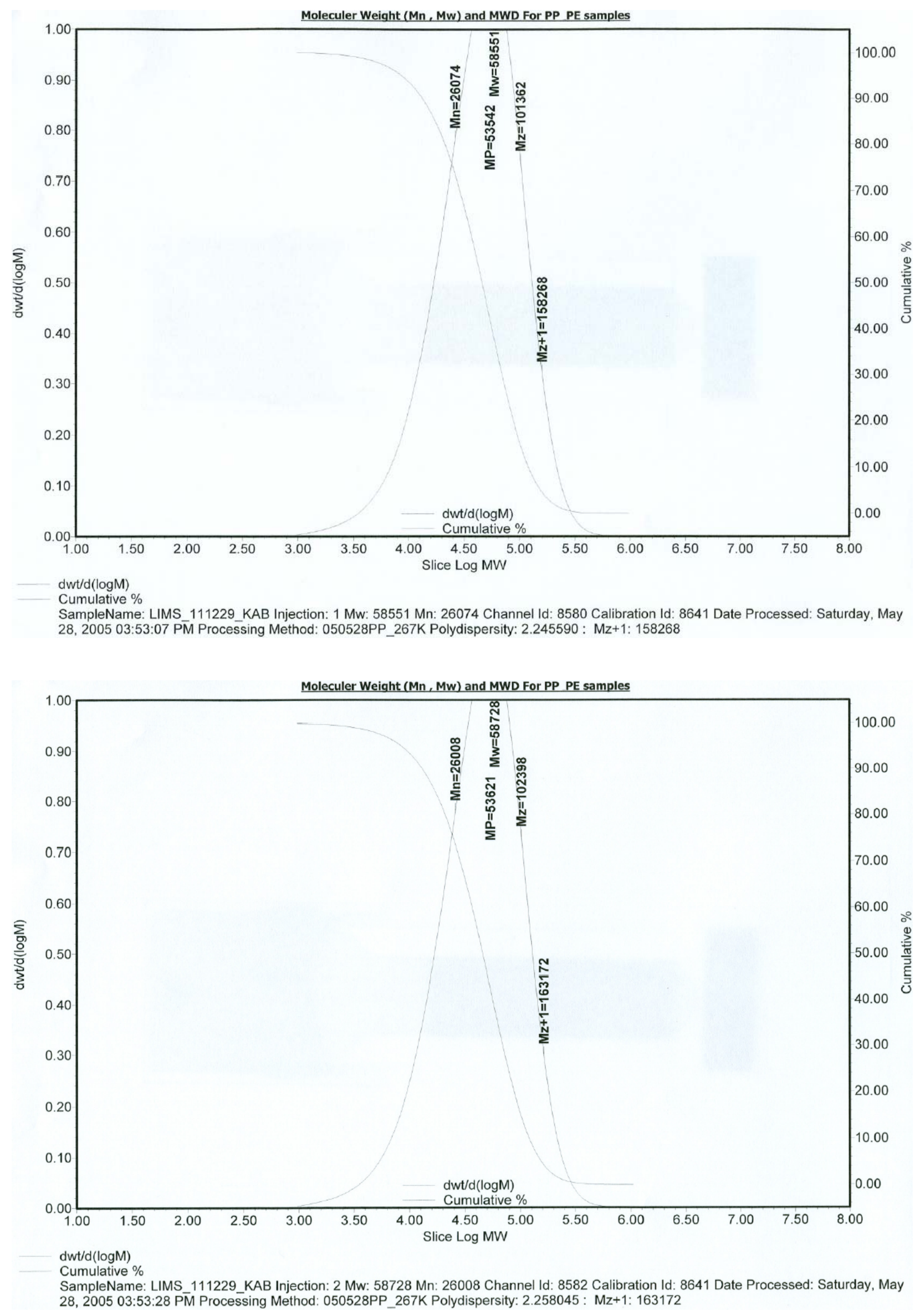
Entry 8: Catalyst 3, $T_{\mathrm{p}}=0^{\circ} \mathrm{C}$
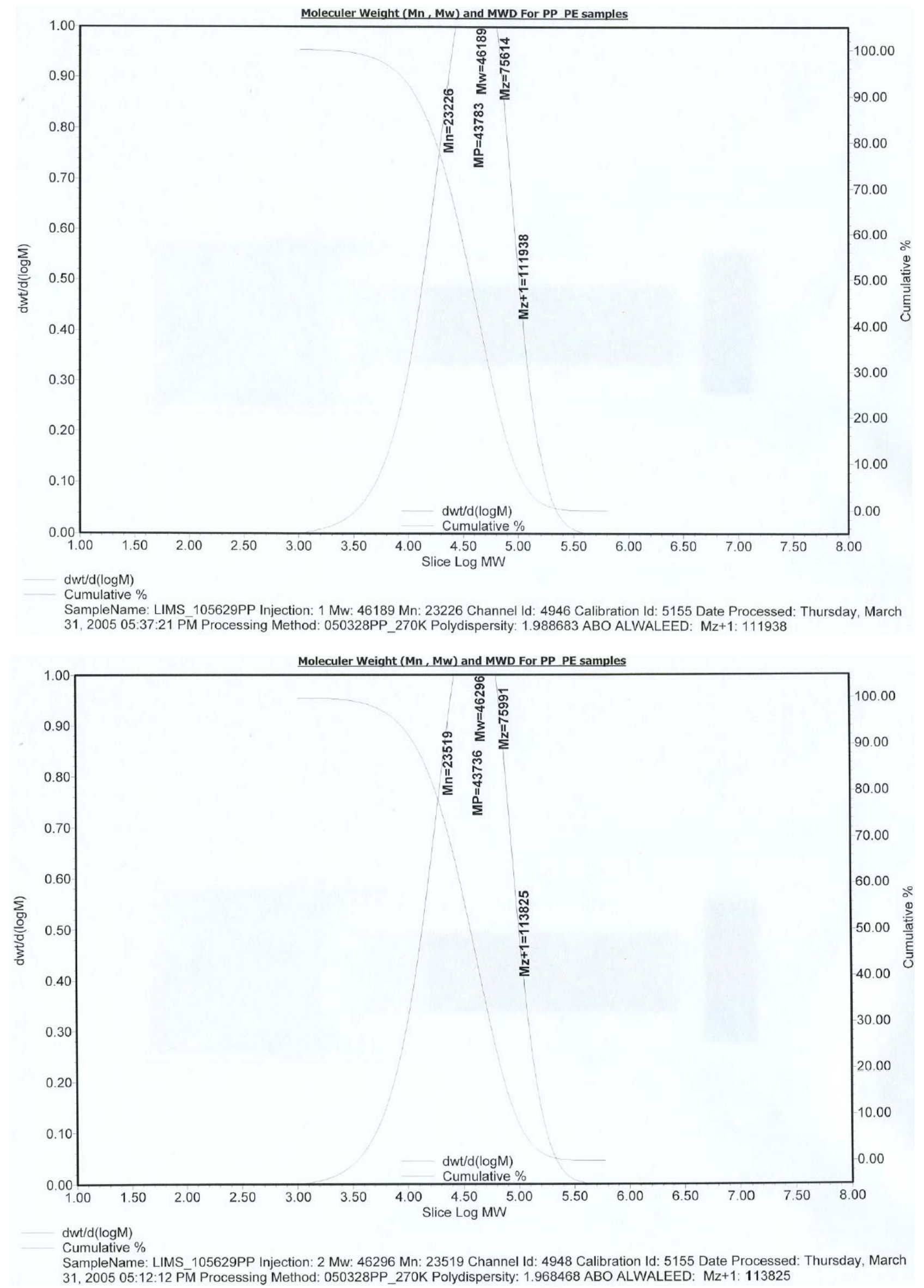
Entry 9: Catalyst 3, $T_{\mathrm{p}}=25^{\circ} \mathrm{C}$

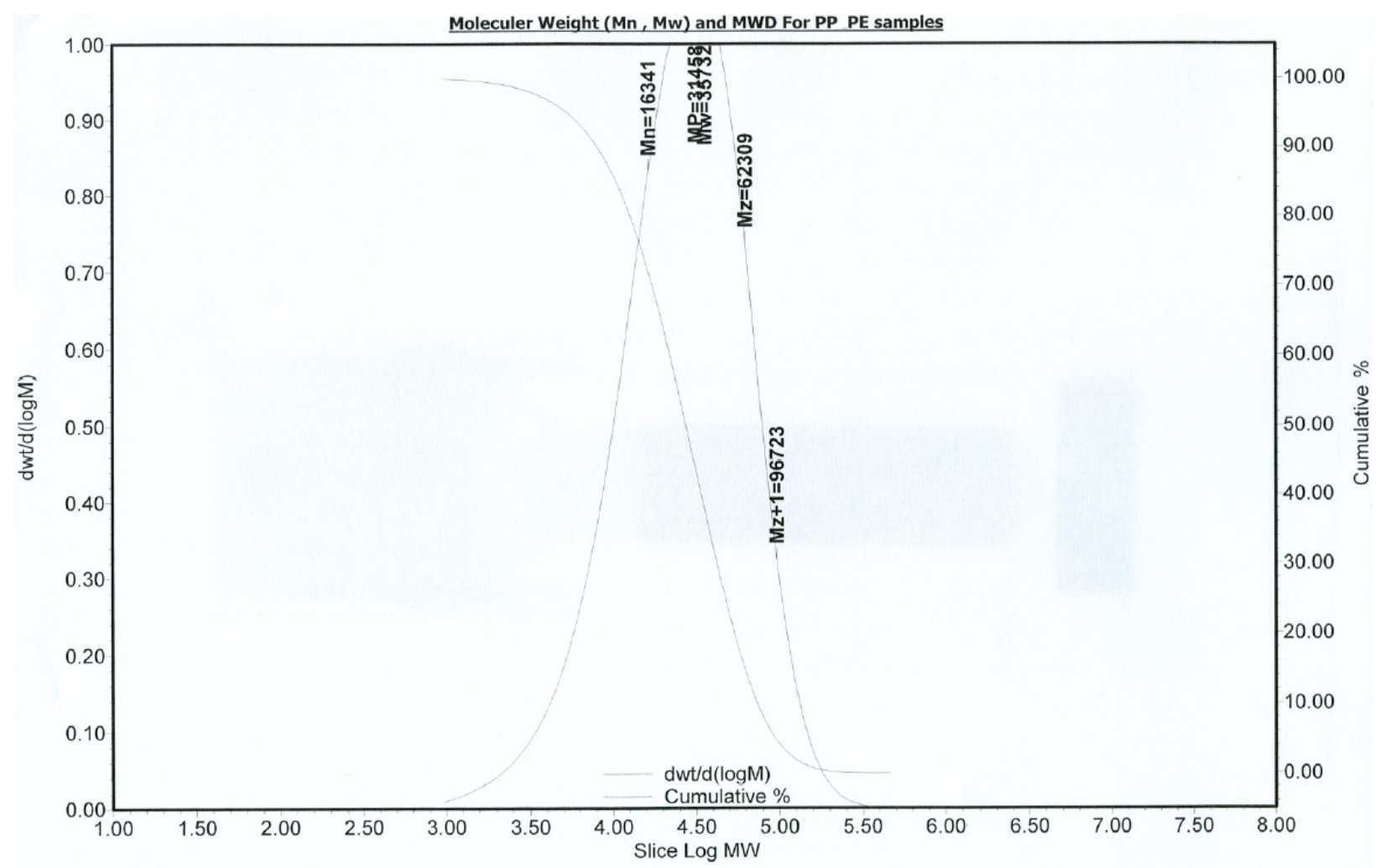

$\mathrm{dwt} / \mathrm{d}(\log M)$

Cumulative \%

SampleName: LIMS 111226 KAB Injection: 1 Mw: 35732 Mn: 16341 Channel Id: 8565 Calibration Id: 8641 Date Processed: Saturday, May 28, 2005 03:51:07 PM Processing Method: 050528PP_267K Polydispersity: 2.186652 : Mz+1: 96723

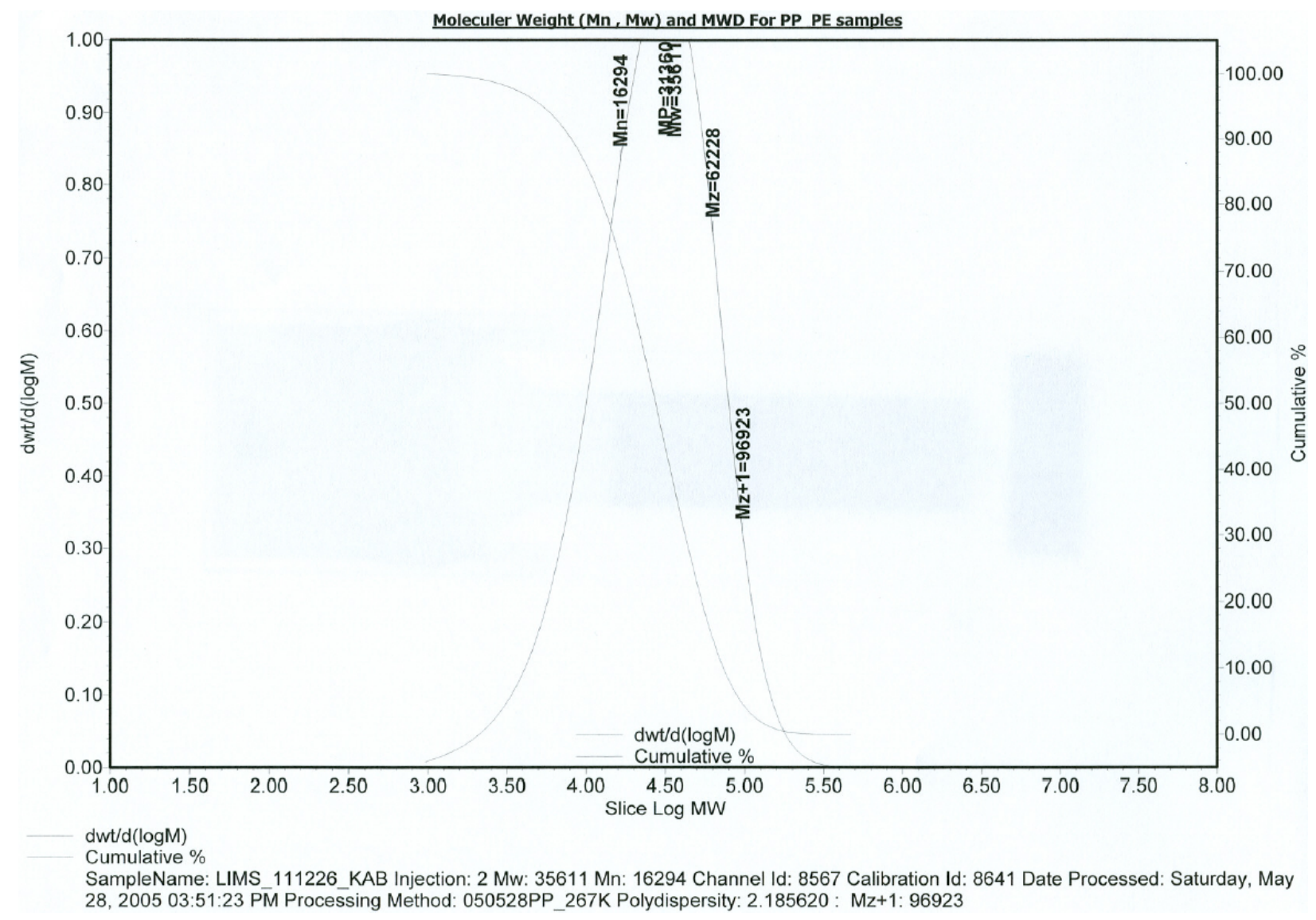


Entry 10: Catalyst 3, $T_{\mathrm{p}}=90^{\circ} \mathrm{C}$
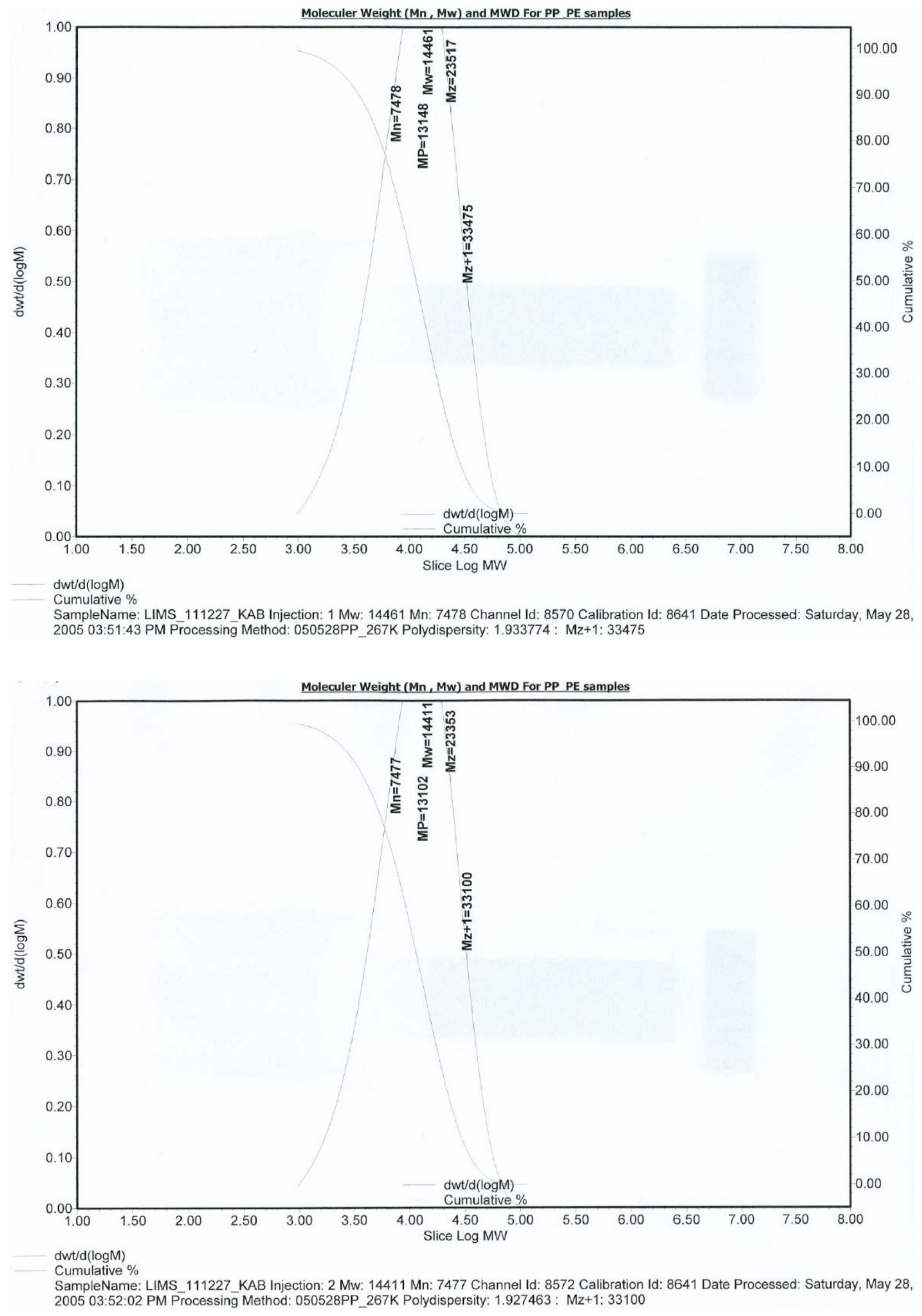
Table 3. Influence of temperature on ethylene homopolymerizations with 3/MAO. ${ }^{a}$

\begin{tabular}{ccc}
\hline Temperature & Yield (g PE) & Activity $^{\mathrm{b}}$ \\
\hline 20 & 0.41 & 490 \\
$50^{\mathrm{c}}$ & 0.74 & 1,330 \\
70 & 3.21 & 3,860 \\
85 & 3.47 & 4,170 \\
100 & 3.68 & 4,420 \\
$120^{\mathrm{d}}$ & 4.25 & 3,830 \\
140 & 2.83 & 3,400 \\
170 & 1.65 & 1,980 \\
\hline
\end{tabular}

${ }^{a}$ Polymerization conditions: $25 \mathrm{~mL}$ toluene, $3.36 \mu \mathrm{mol}$ 3, 1000 eq. MAO, continuous feed of ethylene, 80 psi $(\sim 0.5 \mathrm{M}), 15 \mathrm{~min}$. run. ${ }^{\mathrm{b}} \mathrm{kg} \mathrm{PE} /(\mathrm{mol} \mathrm{M} \cdot \mathrm{h}) .{ }^{\mathrm{c}} 10 \mathrm{~min}$. run. ${ }^{\mathrm{d}} 20$ min. run.

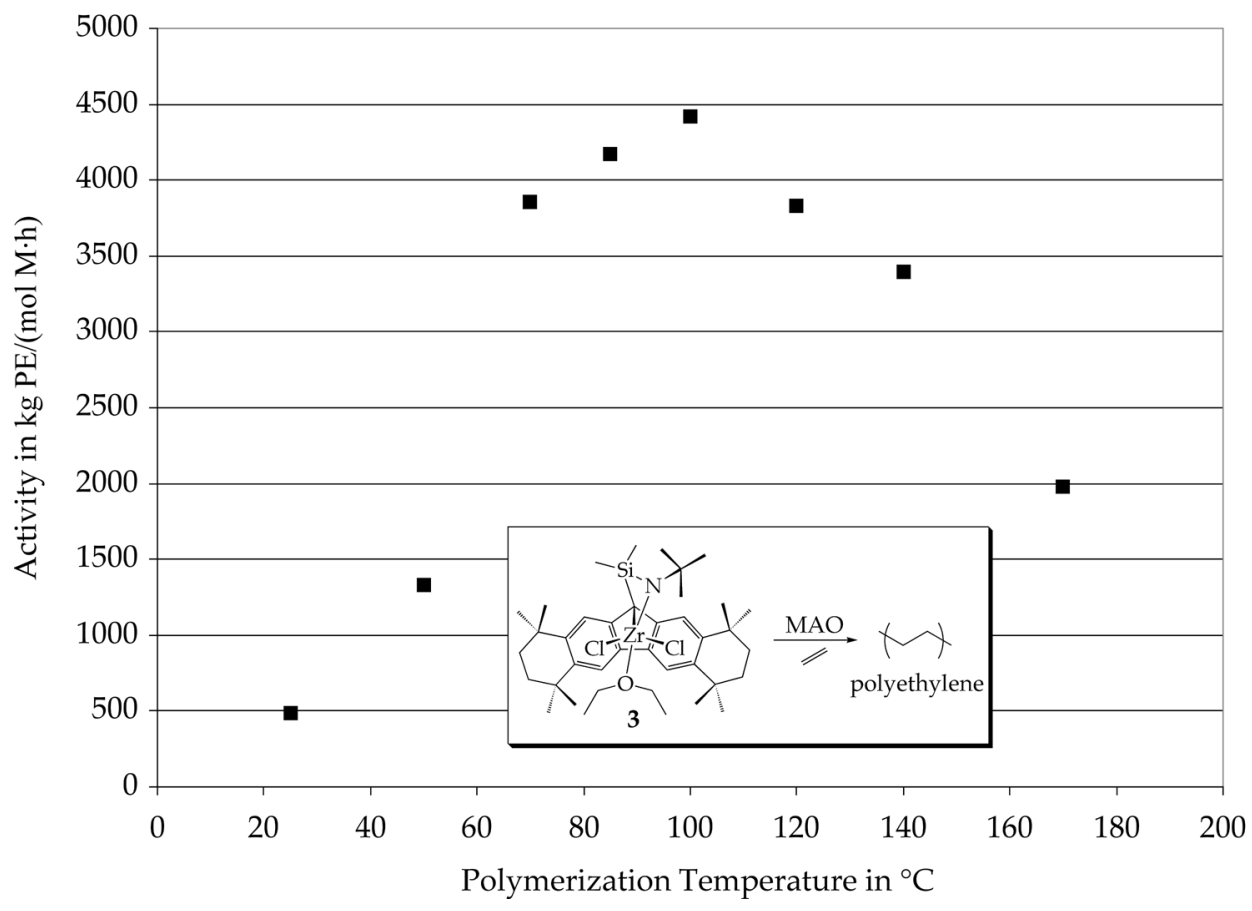

This plot of the ethylene homopolymerization activity of 3/MAO illustrates that the catalyst is active even at $170^{\circ} \mathrm{C}$. It is unclear if the reduction in activity above $100^{\circ} \mathrm{C}$ is due to catalyst decomposition and/or (more likely) the result of reduced ethylene concentration in the solvent. 
Ethylene vs. Propylene Homopolymerization Activity Comparison with 1-5/MAO

Table 4. Ethylene vs. propylene homopolymerization activity with 15/MAO. ${ }^{\text {a }}$

\begin{tabular}{ccrrc}
\hline $\begin{array}{c}\text { Catalyst } \\
(\mu \mathrm{mol})\end{array}$ & Monomer & $\begin{array}{c}\text { Yield } \\
(\mathrm{g})\end{array}$ & Activity $^{\mathrm{b}}$ & $\begin{array}{c}\text { Activity } \\
\text { Quotient } \\
\mathrm{C}_{3} \mathrm{H}_{6} / \mathrm{C}_{2} \mathrm{H}_{4}\end{array}$ \\
\hline $\mathbf{1}(1.62)$ & $\mathrm{C}_{2} \mathrm{H}_{4}$ & 0.06 & 4,820 & 0.16 \\
$\mathbf{1}(1.62)$ & $\mathrm{C}_{3} \mathrm{H}_{6}$ & 0.31 & 790 & \\
\hline $\mathbf{2}(1.53)$ & $\mathrm{C}_{2} \mathrm{H}_{4}$ & 0.02 & 1,400 & 0.87 \\
$\mathbf{2}(1.53)$ & $\mathrm{C}_{3} \mathrm{H}_{6}$ & 0.45 & 1,220 & \\
\hline $\mathbf{3}(1.66)$ & $\mathrm{C}_{2} \mathrm{H}_{4}$ & 0.01 & 460 & \multirow{2}{*}{5.80} \\
$\mathbf{3}(1.66)$ & $\mathrm{C}_{3} \mathrm{H}_{6}$ & 1.07 & 2,670 & \\
\hline $\mathbf{4}(1.66)$ & $\mathrm{C}_{2} \mathrm{H}_{4}$ & 0.08 & 5,860 & 0.14 \\
$\mathbf{4 ( 1 . 6 6 )}$ & $\mathrm{C}_{3} \mathrm{H}_{6}$ & 0.34 & 840 & \\
\hline $\mathbf{5}(1.66)^{\mathrm{c}}$ & $\mathrm{C}_{2} \mathrm{H}_{4}$ & 0.22 & 62,480 & \multirow{2}{*}{0.001} \\
$\mathbf{5 ( 1 . 6 6 )}$ & $\mathrm{C}_{3} \mathrm{H}_{6}$ & 0.04 & 100 & \\
\hline
\end{tabular}

${ }^{\text {aPolymerization conditions: Ethylene: } T_{\mathrm{p}}=25^{\circ} \mathrm{C} ; 1,000 \text { eq. MAO; } 30}$ $\mathrm{mL}$ toluene; 80 psi ethylene $(\sim 0.5 \mathrm{M})$; 10 min. pre-run equilibration; 1 min. run. Propylene: $T_{\mathrm{p}}=25^{\circ} \mathrm{C} ; 1000$ eq. $\mathrm{MAO}$; neat propylene $(30$ $\mathrm{mL}, 14.5 \mathrm{M}) ; 1 \mathrm{~min}$. run. ${ }^{\mathrm{b}}$ Activity expressed in $\mathrm{kg}$ polymer/(mol $\mathrm{M} \cdot \mathrm{h} \cdot\left[\right.$ monomer]). ${ }^{\mathrm{c}} 20 \mathrm{psi}$ ethylene $(\sim 0.125 \mathrm{M})$. 